\begin{abstract}
Title of Document:

EVALUATION OF A CULTURALLY INCLUSIVE MODEL OF SEXUAL MINORITY IDENTITY FORMATION
\end{abstract}

Cristina M. Risco, M. A., 2008

Directed by:

Professor Ruth E. Fassinger

Department of Counseling and Personnel Services

In the current work, the reliability and validity of a measure of sexual minority identity formation (the Same-Sex Orientation Identity Questionnaire; SSOIQ) was assessed with a racially/ethnically diverse sample. The SSOIQ was developed to measure one's location in a sexual minority identity formation process. The measure was derived from the Fassinger and colleagues (McCarn \& Fassinger, 1996)) dual-trajectory model that hypothesizes two separate but reciprocal processes of individual sexual identity development and group membership identity development. Estimates of internal consistency reliability were assessed through Cronbach's alpha. A preliminary evaluation of the theoretical model underlying the measure was conducted by examining the interrelationships of the conceptually distinct phases of the model. Convergent validity was partially established through relationships of the measure to measures of identity confusion, internalized homonegativity, same group orientation, and outness. Discriminant validity was partially established using a measure of dogmatism. 


\title{
EVALUATION OF A CULTURALLY INCLUSIVE MODEL OF SEXUAL MINORITY IDENTITY FORMATION
}

\author{
By \\ Cristina Maria Risco \\ Thesis submitted to the Faculty of the Graduate School of the \\ University of Maryland, College Park, in partial fulfillment \\ of the requirements for the degree of \\ Master of Arts \\ 2008
}

Advisory Committee:

Professor Ruth E. Fassinger, Chair

Professor Emeritus William E. Sedlacek

Assistant Professor Ty D. Tashiro 
(C) Copyright by

Cristina Maria Risco

2008 


\section{ACKNOWLEDGEMENTS}

I would like to acknowledge a number of people who were invaluable to the completion of this thesis:

The participants for their courage and willingness to share their life stories. Much gratitude to all those who so generously helped to recruit participants, their enthusiasm was inspirational.

My committee members, Ty Tashiro and William Sedlacek, for their continued commitment to this project, constructive feedback, and encouragement of me as a scholar.

My advisor Ruth, whose dynamic style as a mentor is truly unmatched. Her confidence in me and belief in this project never failed to energize my spirit. My commitment to research focusing on the needs of the underserved has strengthened tremendously because of her influence.

My family, for the foundation they provide, the sense of pride they instill in me, and their unwavering encouragement throughout this process. Luis Betalleluz, who supported me through every juncture.

Finally, I would like to dedicate this thesis to my uncle Rafael R. Risco and my grandmother Maria P. Risco. It was the coming and going of their lives that provided both the inspiration for this project and the drive to see it through. 


\section{Table of Contents}

Table of Contents

Chapter 1: Introduction

$\begin{array}{ll}\text { Chapter 2: Literature Review } & 7\end{array}$

$\begin{array}{ll}\text { Sexual Orientation and Identity } & 7\end{array}$

Theories of Sexual Minority Identity Development 10

Critique of Existing Models 12

Racial and Ethnic Differences in Sexual Minority Identity Formation 15

Gender Differences in Sexual Minority Identity Formation 22

Models of Multiple Identity Development 28

Inclusive Model of Sexual Minority Identity Formation $\quad 30$

Statement of the Problem $\quad 34$

Hypotheses 36

Chapter 3: Methods $\quad 41$

Design Statement $\quad 41$

Item Development $\quad 41$

Participants $\quad 44$

$\begin{array}{ll}\text { Measures } & 46\end{array}$

$\begin{array}{ll}\text { Procedure } & 50\end{array}$

Chapter 4: Results

Chapter 5: Discussion $\quad 66$

Summary of Findings and Comparison to Existing Literature $\quad 66$

$\begin{array}{ll}\text { Strengths and Limitations of the Study } & 73\end{array}$

Implications for Research $\quad 77$

$\begin{array}{ll}\text { Implications for Practice } & 79\end{array}$

Appendices

APPENDIX A: Item Revision Worksheet $\quad 81$

APPENDIX B: Card Sort Instructions $\quad 85$

APPENDIX C: Card Sort Handout $\quad 88$

APPENDIX D: Recruitment Advertisement $\quad 93$

APPENDIX E: Informed Consent $\quad 94$

APPENDIX F: Survey 96

APPENDIX G: Debriefing 107

APPENDIX H: SSOIQ Scoring Instructions 108

$\begin{array}{lr}\text { References } & 109\end{array}$ 


\section{Chapter 1: Introduction}

The psychological literature that explores sexual minority orientations has evidenced a dramatic shift over the past three decades (Croteau, Bieschke, Fassinger, \& Manning, in press). This shift is indicated by a considerable growth in the literature that examines sexual minority orientations from affirmative perspectives and highlights the significance of unique social tasks faced by sexual minority persons (e.g., Fassinger \& Arseneau, 2007; Fassinger \& Miller, 1996; McCarn \& Fassinger, 1996; Meyer, 1995; 2003).

A primary social task discussed in the literature is that of developing a sense of positive identity within a context of pervasive environmental and internalized homonegativity. Further, understanding oppressive factors that influence the process of developing a positive minority identity is particularly important in light of research that suggests the centrality of one's group identity to mental health and well-being. Meyer $(1995 ; 2003)$ offered a conceptual framework for understanding the prevalence of psychological distress in sexual minority persons. Minority stress theory posits that sexual minority persons in a heterosexist society are subjected to chronic stress related to their stigmatization. Minority stressors are conceptualized as: internalized homophobia, expectations of stigma, experience of prejudice events, hiding and concealing, and ameliorative coping responses. Further, Meyer (2003) suggests that characteristics of minority identity also are related to minority stress and health outcomes. In sum, sexual minority persons transgress privileged heterosexual norms and confront stigmatization that persists across various social institutions (Bohan, 1996; Fassinger \& Arseneau, 2007; Waldo, 1999). These experiences perpetuate a particular struggle with sexual minority 
identity awareness, acceptance, and affirmation as well as the process of self-disclosure (Mohr \& Fassinger, 2000; 2003).

Current models of sexual minority identity development and the coming out process underscore the significance of oppressive contextual influences on normative developmental and psychological processes (McCarn \& Fassinger, 1996; Mohr \& Fassinger, 2000). Sexual identity development has been defined as "the process by which individuals emerge into a psychological sense of themselves that embraces their sexual orientation amidst pervasive societal heterosexism and sexual prejudice" (Croteau et al., in press, p. 7). Thus, conceptualizing sexual minority identity development as a process that begins with confronting assumptions of heterosexuality and heterosexist values further elucidates the fundamentality of oppressive contextual influences to this process.

In an extensive review of the literature, McCarn and Fassinger (1996) examined several well-known theoretical frameworks describing sexual minority identity development (e.g., Cass, 1979; Sophie, 1985/86; Troiden, 1989). In their critique, McCarn \& Fassinger present two primary limitations of well-known sexual minority identity models. The first limitation is that such models imply a common general progression of identity development; yet many have solely conceptualized around the experiences of White men, and the extent to which they incorporate gender, race, and other demographic differences is varied. The second limitation is that most existing lesbian/gay identity models ignore the critical differences between personal and reference group components of identity. These authors concluded that existing models tend to confound two separate developmental trajectories in sexual minority identity formation; an individual sexual identity process involving the recognition and acceptance of a same- 
gender erotic/romantic orientation and a group membership identity process involving the confrontation of oppression and acceptance of one's status as a member of an oppressed social group (McCarn \& Fassinger, 1996; Fassinger, 1991).

McCarn and Fassinger (1996) and Fassinger and Miller (1996) offer a dualtrajectory model, emphasizing the importance of separating the individual process that focuses on sexual awareness and choices from the group process that focuses on group membership and affiliation for sexual minority women and men. The commingling of these two processes implies that individuals cannot be fully integrated in their sexual minority identity in the absence of self-disclosure of their sexual orientation and same gender relationships. What is more, confounding the two distinct processes contributes to the systematic discrimination of racial/ethnic minorities in the assessment of a normative identity process (Fukuyama \& Ferguson, 2000; McCarn \& Fassinger, 1996). Selfdisclosure of sexual minority identity is profoundly influenced by an oppressive context. Further, the degree to which a context is oppressive varies across cultural groups (Bieschke, Hardy, Fassinger, \& Croteau, in press; Smith, 1997). Thus, using selfdisclosure as a marker of positive identity formation potentially discriminates against those who live in more oppressive environments.

Fukuyama and Ferguson (2000) explain that one of the primary limitations of recognizing only single identities is that individuals who embrace multiple identities become invisible members within specific social reference groups. Sexual minority persons of color thus are forced to cope with feelings of visibility or invisibility in at least two communities in which they belong: mainstream sexual minority communities and their respective racial/ethnic communities. Further, psychological identity theories that 
fail to acknowledge concurrent multiple social identities render the complexity of integrating multiple identities and coping with multiple forms of oppression unclear (Fukuyama \& Ferguson). Research exploring the complex experiences of sexual minority persons of color suggests the primacy of the following contexts in the sexual minority identity formation process: family, community, cultural norms/expectations, and oppression. These contexts potentially inhibit the expression, salience, and acceptance of one or multiple identities (Alquijay, 1997; Chan, 1997; Greene, 2000; Smith, 1997). Thus, using a single identity framework to understand the experiences of all sexual minority persons may be ineffective and does not adequately attend to the experiences of the individual integrating multiple minority identities.

In light of these considerations, the current study attempted to further the literature on sexual identity formation by examining the reliability and validity of a sexual minority identity formation measure (reflecting the Fassinger and colleagues dualtrajectory model) with a racially/ethnically diverse population. In this process, the items in two existing versions of the measure (women: Fassinger, 2001a; McCarn \& Fassinger, 1996; men: Fassinger, 2001b; Fassinger \& Miller, 1996) were revised to better capture current understandings of the experiences of sexual minority persons of color. The dualtrajectory model presented for validation was intended to be widely inclusive of the diverse experiences of self-identification in sexual minority persons (both racial/ethnic majority and minority persons). However, because the initial quasi-validation samples were small (and contained so few racial/ethnic minorities), it is unclear whether the measure adequately and accurately captures the experiences of people of color. The current study was part of a sequence of work by Fassinger and colleagues. McCarn and 
Fassinger (1996) first proposed a model of sexual minority identity formation. Empirical support for the model was obtained using a modified Q-sort methodology with a small, diverse sample of lesbian women (Fassinger \& McCarn, 1991; McCarn, 1991). Fassinger and Miller (1996) then developed and provided quasi-validity evidence (again using a modified Q sort methodology) of a comparable instrument for use with gay men. The instruments subsequently were modified (see Fassinger, 2001a, 2001b) based on the findings of Fassinger and colleagues and other researchers. The current study intended to further revise the two versions of the measure and provide evidence of formal validation in a culturally diverse population.

\section{Dual-Trajectory Model of Sexual Minority Identity Formation}

The Fassinger and colleagues (McCarn \& Fassinger, 1996; Fassinger \& Miller, 1996) model described in the current study represents an attempt to address the limitations noted in previous sexual identity models and was intended to be broadly inclusive of the diverse paths one may take to an integrated and synthesized sexual minority identity. The model represents the first sexual identity development model to date that provides two independent trajectories, one involving increased positive identification with one's own internal sense of same sex attraction and the other involving increased identification with a sexual minority reference group (e.g., lesbian, gay, bisexual). The authors proposed a four-phase model with two parallel branches that separate the internal from the sociopolitical process: individual sexual identity and group membership identity, respectively. An initial phase of nonawareness precedes both branches of the model, and the four subsequent phases (awareness, exploration, deepening/commitment, internalization/synthesis) follow in the same progression for 
each branch. Thus, the model explored in the current study represents eight locations in the identity formation process (four phases of identity within each branch). The current study was based on this basic dual-trajectory model, and explores the existing measures representing the model. More specifically, the current study sought to validate revised, updated versions of the existing measures using a sample of sexual minority people of color. The model has been noted recently for its inclusiveness (e.g., Firestein, 2007; Liddle, 2007; Potoczniak, 2007), as well as for its applicability in counseling practice (e.g., Bieschke, Paul, \& Blasko, 2007). Thus, psychometric work specifically investigating its appropriateness for sexual minority people of color contributes to the usefulness of this model (and associated measures) with diverse populations and expands the literature on sexual minority identification. 


\section{Chapter 2: Literature Review}

The psychological literature exploring the process by which sexual minority persons achieve an integrated sense of sexual identity is fairly extensive at this point in time. In this review of the literature, the concepts of sexual identity and selected theories on the sexual minority identity formation process are reviewed. Considerable attention is given to understanding how race/ethnicity and gender may influence the formation of a sexual minority identity. Next, theoretical models integrating multiple aspects of social group identities are discussed. Finally, the Fassinger and colleagues (Fassinger \& Miller, 1996; McCarn \& Fassinger, 1996) dual-trajectory model of sexual minority identity formation is considered as a framework sensitive to the integration of multiple identities.

\section{Sexual Orientation and Identity}

Researchers describe sexuality within three primary domains; sexual orientation, sexual behavior, and sexual identity (Savin-Williams, 2005). Sexual orientation typically is conceptualized as the predominance of erotic/romantic feelings, thoughts, and fantasies one has for members of a particular sex, both sexes, or neither sex. Considerable attention is given in both the scholarly and public discourse to the origins of sexual orientation. Debate between biological (e.g., genetic, hormonal, physiological) and environmental (e.g., psychogenic, social) explanations typically has characterized such discussions (Bohan, 1996, Savin-Williams, 2005). However, most contemporary theorists view sexual orientation as much more complex and have moved beyond polarized discussions of nature versus nurture. Such scholars acknowledge the possibility of biological influences on the development of sexual orientation and consider evidence pointing to the 
social construction of sexual orientation as an identity (see Fassinger, 2000; Fassinger \& Arseneau, 2007).

Sexual identity refers to a socially recognized label that represents sexual behaviors, erotic fantasies/attractions, patterns of sexual and emotional arousal, affectional and intimate preferences and attachments, gender identity, social sex-role, lifestyle, community, self-disclosure, political allegiances, and self identification (e.g., Fassinger \& Arseneau, 2007; Gonsiorek, 1995, Mohr \& Fassinger, 2000, Peplau, 2001; Savin-Williams, 2005). From a postmodern feminist perspective (Enns, 2004), individuals make use of labels to capture the way in which their identity is organized in a particular temporal and situational context (Fassinger \& Arseneau, 2007). Moreover, individuals are limited to the identities defined by their particular cultural and temporal context. Thus, sexual identities are not universal nor are they resistant to change. In a Euro-American context, an individual's sense of personal identity as gay, lesbian, bisexual, or heterosexual often is assumed to be a core element of sexual orientation. However, same-sex attractions and relationships are not inevitably linked to identity. Indeed, many individuals report correspondence between sexual orientation, behavior, and identity but patterns of non-correspondence are common and frequently noted in the literature (e.g., Diamond \& Savin-Williams, 2000; Fassinger, 2000; Fassinger \& Arseneau, 2007; Savin-Williams, 2005). At times, identity may be partial, contradictory, or strategic (Enns, 2004).

A lack of one-to-one correspondence between sexual orientation, behavior, and identity implicate limitations in the measurement of sexual identity, particularly regarding the use of labels. However, sexual identity formation frameworks remain a 
useful tool for practitioners and researchers. The way one comes to label her- or himself is considered to be a relatively important aspect of self in contemporary America. For this reason, practitioners and researchers are interested in how the individual comes to these processes developmentally.

The model on which the instruments tested in this study are based is intended to capture one's current location in a recursive identity formation process (McCarn \& Fassinger, 1996; Fassinger \& Miller, 1996). For example, three women may similarly endorse experiences reflective of the integration/synthesis phase of the model. However, one woman may self label as lesbian, another as queer, and the third as bisexual. In this example, what is represented by all three of these women is a positive and affirmed identity (organized around same-sex attraction) that has been incorporated successfully into the overall self-concept. The particular label each woman associates with her identity is a symbolic representation of her understanding of her sexual feelings, attractions, and behavior. In the current model, the authors consider the experiences of those who have some component of same sex attraction and/or orientation represented in their sexual identity. It should be noted that the particular label that one attaches to his or her sexual minority identity is not under inquiry. For this reason, the proposed measure for validation is referred to as the Same-Sex Orientation Identity Questionnaire (SSOIQ). The term same-sex orientation identity is thought to be inclusive of the various labels individuals associate with their identities (Croteau et al., in press). Lesbian women, gay men and women, bisexual men and women, queer persons, and other labels indicating same-sex orientation or attraction are considered together due to similar developmental experiences resultant from an oppressive context (Fassinger \& Arseneau, 2007). 


\section{Theories of Sexual Minority Identity Development}

Sexual minority identity development models first emerged in the psychological literature of the 1970s. Such models conceptualize how individuals integrate new aspects of awareness within a core sense of self and typically focus on aspects of identity associated with minority status, marginalization, or oppression (Enns, 2004). The majority of these models posit that individuals move through stages (or phases) that are initially characterized by internalized stigmatization or a lack of recognition or salience with regard to a particular sexual minority identity. Over time, individuals become increasingly aware of an oppressive context and often experience periods of disruption and questioning. Later phases are generally characterized by cognitive flexibility, selfdefinition, and affirmation (e.g., Cass, 1979).

Initially, most models of identity development proposed a somewhat linear path that focused on one aspect of identity such as gay identity (e.g., Cass 1979), or lesbian identity (e.g., Sophie, 1985/86). More recently, however, theorists and researchers have shifted in their thinking about identity development and suggest that identity may not follow a linear pathway, particularly when individuals are negotiating multiple aspects of identity that may be associated with different levels of privilege and/or oppression (Enns, 2004).

What follows is a brief review of two sexual minority identity development frameworks that each represent a particular approach to understanding identity formation; the multistage and lifespan approaches [Cass (1979) and D'Augelli (1994) respectively]. There are numerous models that have been presented in the literature (e.g., Coleman, 
1982; Minton \& McDonald, 1984; Sophie, 1985/1989; Troiden 1989); however only two are described here in detail as they typify the way such models are conceptualized.

Since its development, the Cass (1979) model represents the most frequently cited theory and one of the few that has been tested empirically (see Cass, 1984), albeit with limitations. Further, this model has formed the foundation for a majority of the work on sexual minority identity formation (McCarn \& Fassinger, 1996). Cass presented a model of homosexual identity formation based on interpersonal congruency theory. The model assumes that the acquisition of a homosexual identity is a developmental process resulting from the interaction between the individual and his or her environment. Cass originally presented six stages of perception and behavior (later revised to four stages), moving from minimal awareness and acceptance of a homosexual identity to a final stage in which homosexual identity is integrated with other aspects of self. Prior to the first stage, individuals perceive themselves as heterosexual. As one's perceptions change, increased conflict occurs between self-concept, behavior, and the perceptions of others. This conflict results in one's movement through six developmental stages; Identity Confusion, Identity Comparison, Identity Tolerance, Identity Acceptance, Identity Pride, Identity Synthesis. Cass's stages have both a cognitive component reflecting how individuals view themselves and an affective component indicating how they feel about their own and others' perceptions. Motivation for development is viewed as the need to resolve the incongruence that each stage creates interpersonally and in reference to society. Individuals work through each stage, remain at a particular stage, or undergo identity foreclosure, terminating forward movement in the homosexual identity formation process. Also, Cass (1984) indicated that sex-role socialization might result in differences 
in how women and men negotiate the developmental process and suggested that societal attitudes at different historical periods would influence how the individual approached identity formation.

In contrast, D’Augelli (1994) presented a life span model of lesbian, gay, and bisexual identity development that considers the complexity and idiosyncrasy of the individual developmental process and how this process varies with context over time. D’Augelli argued against the essentialist notion of earlier identity development models (e.g., Cass, 1979) that hold that identity is formed in sequential stages, achieved by early adulthood, and then endures throughout life. Instead, identity is viewed as a social construction, shaped to varying degrees by social circumstances and environment and malleable throughout life. At certain times, sexual identity may be very fluid, whereas at other times, it may be more solidified. Hormonal changes, social circumstances, and peer relationships at different life stages are three factors that may influence developmental plasticity.

D’Augelli (1994) introduced six interactive processes (as opposed to stages) involved in lesbian, gay, and bisexual identity development: Exiting Heterosexual Identity, Developing a Personal Lesbian/Gay/Bisexual Identity Status, Developing a Lesbian/Gay/Bisexual Social Identity, Becoming a Lesbian/Gay/Bisexual Offspring, Developing a Lesbian/Gay/Bisexual Intimacy Status, Entering a Lesbian/Gay/Bisexual Community.

\section{Critique of Existing Models}

Criticisms of sexual minority identity development frameworks (e.g., Cass, 1979; Coleman, 1982; Minton \& McDonald, 1984; Sophie, 1985/86; Troiden, 1989) have been 
broad and generally provide three key limitations (e.g., Savin-Williams, 2005). First, multistage models of sexual minority identity development (e.g., Cass, 1979; Sophie, 1985/86) have been critiqued for their assumption of an inherent developmental process, a process that is universal across time and context. Such models are assumed to undermine the complexity of the individual experience and the diversity within the sexual minority community. Identity theorists have asserted that progression through all stages (or phases) is not a universal experience and emphasized that individuals make choices and play an active role in the development of their identities (e.g., McCarn \& Fassinger, 1996). Theorists also have highlighted that the developmental process is largely influenced by social factors (McCarn \& Fassinger, 1996; Troiden, 1989). Further, D'Augelli (1994) along with other identity theorists (McCarn \& Fassinger, 1996) have cautioned that identity development models are not intended to describe a uniform experience and expressed an appreciation for the complexity of individuals and context. A key concept that D' Augelli borrows from the lifespan perspective is the idea of individual difference. That is, no two individuals follow the same developmental trajectory. D'Augelli suggests that there may be more similarities in sexual self-definition in certain periods in life, such as late adulthood; in certain kinds of families, such as those not valuing difference; in certain communities, such as those that are highly homogeneous; and in certain historical periods, such as the late 1950s.

A second critique of existing models concerns the lack of empirical evidence that support such frameworks (e.g., Coleman, 1982; D’Augelli, 1994; Troiden, 1989). A majority of the early theories of homosexual, gay, lesbian, or bisexual identity development were based on interviews that inquired about respondents' experiences in 
their sexual minority identity formation process (e.g., Coleman, 1982). Few, if any, standardized measures of theorized developmental sequences were developed to allow for validation of the models. Cass (1984), however, attempted to assess the validity of her model by developing two measures, the Stage Allocation Measure (SAM) and the Homosexual Identity Questionnaire (HIQ). Though her findings suggest a general correspondence between the two instruments, some stages on the HIQ were identified more clearly than others. Also, the methodological underpinnings of Cass's work are questionable in that participants self-designated their stages in the SAM. Further, the validation studies consisted of Australian samples and have been generalized to persons in the United States. More recently, Johns \& Probst (2004) did not find support for the Cass model of discrete stages. Data revealed that participants viewed the identity formation process as best described by two stages; unintegrated versus fully integrated. No measure exists for D'Augelli's model of gay, lesbian, and bisexual identity development (1994). D’Augelli emphasized the importance of using multiple measures to assess each of the factors that influence development across the life span and advocated the use of longitudinal studies to investigate development over time.

A third criticism is that sexual identity models are particularly negligent with regard to gender, racial/ethnic, and cohort differences (Croteau et al., in press; McCarn \& Fassinger, 1996). Profound political awareness (e.g., Cass, 1979) and self-disclosure (e.g., Cass, 1979; Coleman, 1982) are conceptualized as a requirement for identity integration. "Progress" in these models is measured in terms of movement along a continuum that has been conceptualized largely around the experiences of White gay men (Parks, Hughes, \& Mattews, 2004). However, the process of sexual minority 
identification is characteristically more varied and fluid for women relative to men (Peplau, 2001). Clearly demarcated boundaries between lesbian women, bisexual women, and heterosexual women overlook the complexity and ambiguity of women's sexualities. Further, racial/ethnic minorities are profoundly influenced by specific cultural, class, and sociohistoric contexts and sexual identity is continually negotiated across contexts. What follows is a more in depth discussion of the diverse experiences of racial/ethnic minority persons in the sexual minority identity formation process.

\section{Racial/Ethnic Differences in Sexual Minority Identity Formation}

Researchers have noted that the unique cultural experiences of racial/ethnic minority persons rarely have been incorporated into theories and models of sexual identity development (Croteau et al., in press; Fukuyama \& Ferguson, 2000; Harper, Jernewall, \& Zea, 2004; Parks et al., 2004). Previous research on sexual minority identity development has been conducted on predominantly White, middle class samples of older gay men (Parks et al., 2004). The following sections discuss four specific challenges confronted by sexual minority individuals of color in the identity formation process. First, sexual minority women and men of color encounter racism within the mainstream sexual minority community (Chung \& Katayama, 1996; Savin-Williams, 1996). Second, sexual minority people of color experience conflicting cultural values and homonegativity as a sexual minority individual within their racial/ethnic communities (Chan, 1995; Greene, 2000; Holmes, 2001). Further, challenges stemming from competing identity groups potentially impede the desire to develop an integrated identity (Alquijay, 1997). Finally, sexual minority persons of color must negotiate and manage identity across contexts (Zea, Reisen, \& Diaz, 2003). 


\section{Racism in the Mainstream Sexual Minority Community}

Sexual minority persons of color experience racism and discrimination within the context of the sexual minority community through interpersonal interaction with peers as well as systemic experiences (Holmes, 2001). Both forms of racism/discrimination perpetuate a particular struggle for the development of a positive sexual minority identity.

White majority persons may not consider race a salient identity unless the power and privilege associated with their majority status are called into question (Helms, 1990, 1992). In contrast, there are consequences for the racial/ethnic minority person who does not acknowledge his or her cultural background (Smith, 1999). Manalansan (1994), for example, interviewed 50 gay Filipino men in New York City to discover more about their experiences as gay men of color. Many of the men who perceived discrimination suggested that their experiences were largely influenced by societal perceptions of Asian immigrants. This belief was consistent for both immigrant and US born participants. In an exploratory study of six Black gay men and lesbian women, Loiacano (1989) found that participants experienced difficulty in creating a discourse on race with their White majority peers. Instead of feeling supported by the gay and lesbian community, these individuals felt excluded and less supported than their White peers. Loiacano further noted that gay and lesbian groups continue to marginalize Black members while providing more affirmation for White members. Smith (1999) suggested that the lack of

support for racial/ethnic minorities in the gay community is a result of the socialization of White majority persons to appreciate their privileges, either consciously or unconsciously. 
Savin-Williams (1996) put forward that to be gay or lesbian and a racial/ethnic minority means having to expect racism and race-based discrimination within the community one turns to for support. As a result of perceived racism in the sexual minority community, racial/ethnic minority persons may either prefer sexual minority organizations that solely support people of color or withdraw from active participation within such communities. The process of determining the best community of fit necessitates additional developmental tasks for sexual minority persons of color.

\section{Conflicting Values}

Not only do sexual minority persons of color encounter discrimination from the mainstream sexual minority community, but they also may experience difficulty feeling connected to their racial/ethnic communities (Bieschke et al., in press; Holmes, 2001). The challenges encountered by these individuals partly derive from the competing values of their particular racial/ethnic culture and sexual minority culture (Cohen \& Jones, 1999; Holmes, 2001). The values and norms regarding gender, sexuality, and sexual orientation particular to each racial/ethnic group present unique tasks in developing a positive sense of individual and group oriented sexual identity (Bieschke et al., in press).

Racial/ethnic minority persons may experience less flexibility in developing their sexual identity than their majority peers. For example, in contrast to Western expectations favoring individuality, a strong sense of community and emphasis on family privacy may limit Asian American gay men and lesbian women from meeting their personal needs for same-sex intimacy (Chan, 1995). An additional challenge is that homonegativity in an individual's racial or ethnic community may be more pervasive than in the majority White culture (Savin-Williams, 1996). Homonegativity often is justified by religious 
beliefs, leaving sexual minority individuals feeling unsupported within their racial/ethnic communities (Greene, 2000). Thus, sexual minority persons of color oscillate between confronting racism in the sexual minority community and accentuated homonegative notions of sexuality in their particular cultural community. This creates a complex environment for growth and development that can feel overwhelming for sexual minority persons of color. This experience is further intensified in the absence of an appropriate amount of social support.

A critical aspect of racial socialization that takes place within the context of family involves learning to navigate through oppressive racial barriers. However, in the same familial context, learning how to navigate through homonegativity may not be possible (Greene, 2000). Thus, sexual minority persons of color must extend beyond their families to learn such skills and obtain social support. However, given that sexual minority communities may be racist, it becomes difficult for the sexual minority person of color to obtain the support needed to facilitate positive identity development.

\section{Conflicting Allegiances}

Akerlund and Chung (2000) summarized the quandary of sexual minority women and men of color as individuals who are "faced with a unique challenge-integrating two identities, one pertaining to ethnic culture and the other to sexual orientation, in a society that does not fully accept either one" (p.280). Moreover, Greene (1997) noted that for lesbian women and gay men of color, disclosing a lesbian or gay sexual identity may be perceived as disloyal to the culture and community.

Accompanying the development of a sexual minority identity is the challenge of determining what cultural values from which to understand and explain one's experience 
(Holmes, 2001). Possible cultural pressures including centrality of family, traditional gender roles, religious values, and pervasive homonegativity implicate a particular struggle for sexual minority people of color in an identity integration process (Loicanao, 1989; Martinez \& Sullivan, 1998; Savin-Williams, 1996; Smith, 1997). At times, choosing culturally based values is associated with being less affirming of sexual minority values and vice versa (Chung \& Katayama, 1996). Alquijay’s (1997) study on Latina lesbian women in Southern California illustrates this argument. The participants were administered several surveys assessing self-esteem, cultural life style, and homosexual identity formation. Using the Homosexual Identity Questionnaire, Alquijay found that cultural resistance, the ability to actively or passively refuse to adopt cultural norms of the host culture, significantly predicted women who were in the identity confusion stage as defined by Cass' $(1979,1984)$ framework. While the sample cell sizes were small, the findings indicated that Latina lesbian women with higher resistance to United States culture were more likely than other women to represent an earlier stage of identity development. A limitation of this study was that resistance to United States culture was not considered a legitimate refusal of norms given the complicated sociopolitical history between the United States and Latin America. This limitation not withstanding, the findings provide an illustration of conflicting allegiances.

\section{Negotiated Identities}

For sexual minority individuals of color, the pressure to choose one community over another may also lead to a disjointed sense of self. Chan (1997) suggested that Asian American lesbian women experience anxiety over having to choose a cultural community. The apprehension stems from the view that choosing a lesbian identity will 
lead to others identifying them solely by their sexuality while overlooking equally important and meaningful aspects of their identity.

Differential treatment in both the racial/ethnic minority community and the predominantly White sexual minority community may lead some sexual minority persons of color to conceal aspects of their identity, depending on the context of their interactions. Often sexual minority persons of color with fully integrated identities must negotiate aspects of their various identities across contexts. Zea et al. (2003) illustrated this in their finding that some Latino men identify as gay when they are within the context of a gay bar but not when they are with their families. These findings illustrate that sexual minority people of color experience varying degrees of visibility within their own communities (Fukuyama \& Ferguson, 2000). Wilson and Miller (2002) examined heterosexism management in a sample of 37 African American gay and bisexual men. Using a grounded theory approach, five strategies regarding sexual identity management were identified. In non affirmative contexts, participants reported gender role-flexing, reliance on spiritual faith, openly confronting heterosexism, and abstaining from samesex behaviors as strategies for encountering stigma or initiating social change. This study suggests that, prompted by context, various identity management strategies are utilized by sexual minority men of color.

Rosario, Schrimshaw, and Hunter (2004), in a longitudinal study of 145 lesbian, gay, and bisexual youths, found support for the notion that cultural factors do not impede the sexual minority identity formation process but may delay identity integration. Black youths, when compared to White youths, reported less involvement in gay related social activities, reported less comfort with disclosing their sexual identity to others, and thus 
disclosed their identity to fewer people. Also, Latino youths disclosed their identity to fewer people relative to White youths. Analyses of change indicated that Black youths had greater increases in positive attitudes towards homosexuality and in certainty of their sexual identity over time than did White youths. These findings suggest that the process of identity integration is somewhat delayed and not directly linked to disclosure for Black and Latino youths when compared to White youths.

Researchers have suggested that cultural pressure biasing heterosexuality lead many racial/ethnic minority individuals to a bisexual orientation or bisexual behavior and to identify as bisexual rather than gay or lesbian (e.g., Smith, 1997). However, there exists only partial support for this position in the available research. In studies of women (Morris \& Rothblum, 1999) and male youths (Dubé \& Savin-Williams, 1999), no racial/ethnic differences in sexual orientation (defined as erotic attractions for or fantasies about the same or other sex) or in sex of sexual partners were found. Yet, studies of sexual identity continually support significant racial/ethnic differences (e.g., Parks et al., 2004). These findings suggest that cultural pressures may have little influence over sexual orientation or sexual behavior, although such pressure may affect sexual identification.

Savin-Williams (2005) suggests that there is substantial variability across cultures in definitions of and attitudes toward same-sex orientations and same-sex relationships. Further, Euro-American definitions of sexuality are exceedingly rigid relative to those of cultures where sexual identity labels are scarce. In some nonwestern cultures, one's identity is not defined by sexual behavior or the sex of the partner with whom one engages in a sexual and/or romantic relationship. Further, because the public discourse in 
such cultures is less concerned with sexuality as other social identities may be more salient (e.g., religious, regional, ethnic), individuals within these cultures typically do not organize a formal identity around sexuality. Thus, sexual identity developmental frameworks that center on the social group or political aspects of sexual identity may be irrelevant to the individual from a non Euro-American context who integrates components of same-sex orientation.

\section{Summary}

Sexual minority persons of color face common challenges including; discrimination due to race/ethnicity and sexuality, having to choose one identity over another, negative reactions from close friends and family, and the absence of a salient community to turn to for support (Akerlund \& Cheung, 2000). Given this context, racism, sexism, and heterosexism must be confronted in both the dominant and minority cultures in order to progress toward and integrated identity (Greene, 2000). Sexual minority persons of color confront a difficult choice regarding their allegiances (Holmes, 2001). Indeed, the pull to choose one aspect of identity at the risk of rejecting another presents a difficult decision with few resolutions. Thus, perspectives on sexual minority identity development that are sensitive to these struggles more accurately capture how one arrives at an integrated and holistic self.

\section{Gender Differences in Sexual Minority Identity Formation}

Gender differences in sexuality have been widely documented and persist regardless of sexual orientation (Chivers, Rieger, Latty, \& Bailey, 2004). Peplau (2001) provides a paradigm for understanding women's sexual orientation that highlights the centrality of love and intimate relationships as a context for women's sexuality. What 
follows is a discussion of gender differences in sexual identity development rooted in institutionalized sexism and differential socialization experiences. Lesbian models of sexual identity development then are reviewed briefly. Finally, specific attention is given to women of color, as their experiences rarely have been incorporated into developmental frameworks.

\section{Sexism in the Mainstream Sexual Minority Community}

The predominant culture of institutionalized sexism permeates through the sexual minority community. Andersen and Hill Collins (1998) assert that sexism persists in social behaviors and is embedded in cultural symbols, further fortifying gender inequality in social institutions. This renders the individual with a same-sex orientation as one who challenges male-dominated heterosexual norms and the culture of institutionalized sexism.

For women with same-sex attractions, male-dominant views of sexuality represent a difficult barrier to constructing an affirmative lesbian identity. Akerlund and Cheung (2000) conducted a review of literature pertaining to sexual minority people of color over a ten-year period. In their review, these authors suggested that women who identified as lesbian, relative to men who identified as gay, experienced more discrimination from their racial/ethnic community as well as the sexual minority community. This finding was interpreted in the context of the low status of women on the gender hierarchy. Male dominance and adherence to traditional gender roles is persistent in communities of color, particularly for African American and Latino cultures (González \& Espín, 1996). Gender Identity and Socialization Experiences 
Distinct socialization experiences for men and women perpetuate beliefs about appropriate gender behavior, particularly in regard to sexuality (McEwen, 1996). These belief structures influence how sexual minority women and men manage issues such as same-sex relationships, the formation of their sexual identity, and self-disclosure. For example, a lesbian woman's political beliefs may be central as she develops her sexual identity (Brown, 1995) while this may be less critical for her gay male counterpart.

Literature suggests that women have a more relational or partner-centered orientation to sexuality and men a more recreational or body-centered orientation (Peplau, 2001). Gonsiorek (1995) noted that men are socialized to view relationships as competitive and autonomous. Women, on the other hand, are encouraged to develop and express intimacy with others. Consequently, relationship difficulties for women typically center on issues of autonomy and individuality while men may tend to exhibit difficulty with intimacy.

Another aspect of sexual identity formation influenced by distinct socialization experiences concerns the process of coming out. Gonsiorek (1995) has suggested that coming out may be a more fluid process for women given the greater flexibility women are permitted in emotional expression and relationships with other women. For men, however, interaction with other men is much more prescribed according to rigid standards of masculinity that accentuate heterosexuality. For this reason, men may experience greater psychological distress as they develop and disclose a same-sex orientation (Meyer, 1995).

The context of coming out also may be influenced by gender socialization (Holmes, 2001). Sexual activity during the coming out process may be more central for 
men than for women, and men may be more likely to define and substantiate a sexual minority identity with sexual experiences (Minton \& McDonald, 1984). Alternatively, because women are socialized to be relationally or other oriented, sexuality typically is affirmed and validated in the context of an emotional-romantic relationship (Gonsiorek, 1995). Hence, sexual minority women potentially have more in common with heterosexual women than they do with sexual minority men. The work of Chivers et al. (2004) suggests that women as a group, regardless of sexual orientation, have a similar pattern of sexual arousal. When shown explicit sex films, lesbian and heterosexual women did not differ in their subjective and genital arousal to either male-female or female-female sex scenes, and the highest arousal for both groups of women was to heterosexual sex scenes and the least to male-male sex scenes. By contrast, gay-identified and heterosexual men differed in their pattern of sexual arousal. That is, gay men were more aroused by male-male than male-female scenes, and heterosexual men were more aroused by male-female scenes, yet had a stronger subjective reaction to female-female scenes. These findings speak to the greater fluidity of women's (particularly young women's) sexuality as well as the more rigid boundaries that apply to men's sexuality. Moreover, the gender differences in sexuality discussed here point to the interrelation of gender and sexual orientation and suggest that a measure designed to capture the process of sexual orientation identity development be formulated with these considerations.

A growing body of literature documenting gender differences suggests that current models are inadequate for understanding sexual identity development in women (McCarn \& Fassinger, 1996; Savin-Williams \& Diamond, 2000). Although lesbian women and gay men have reported similar identity milestones (awareness, deciding, and 
disclosure) at somewhat similar ages (Cox \& Gallois, 1996), substantial variability exists between gay men and lesbian women (Savin-Williams \& Diamond, 2000). Taking into consideration the unique and complex interaction between gender and sexuality, models of lesbian identity development have been conceptualized.

Lesbian Models of Sexual Minority Identity Formation

A considerable amount of the existing literature focuses on, and primarily is rooted in, the experiences of gay men and then is expanded to include lesbian women (Eliason, 1996). Given that gender is so closely tied to sexuality, this represents a limited and biased approach to understanding the experiences of how lesbian women develop their sexual identity. Consequently, theories and models specifically developed for lesbian women have emerged (e.g., Chapman \& Brannock, 1987; Faderman, 1984; Morris, 1997, Sophie, 1985/86). Below, the Morris model is discussed as it characterizes the typical approach to conceptualizing lesbian identity formation and represents an attempt to address the limitations of earlier models.

Morris (1997) took a divergent approach from the traditional stage models of lesbian identity formation (e.g., Sophie 1985/86) by describing the process of coming out as multidimensional, involving four interrelated and yet distinct dimensions: Sexual identity formation, disclosure of sexual orientation, sexual expression and behavior, and lesbian consciousness. Each dimension is intended to be sensitive to contextual variables such as race/ethnicity, age, income, education, and geography. With the exception of the Morris model, previous lesbian identity formation models deemphasized the diversity within the lesbian population and the impact of cultural context on the eventual outcome of a lesbian identity. Such models are limited in their applicability to the experiences of 
women whose identity formation process will not result in an explicit lesbian identity. However, the Morris model fails to be inclusive of the experiences of women of color in that markers of movement along the dimension of lesbian consciousness include participation in lesbian communities and identification with feminist attitudes. Women of color may choose not participate in lesbian communities or endorse feminist attitudes, thus markers of developmental advancement for these women may be different.

\section{Sexual Minority Women of Color}

Prior empirical research on sexual minority persons of color rarely has included an exclusive focus on the experiences of women (Greene, 2000; Harper et al., 2004). Given the gender-based oppression that women of color often face owing to pervasive sexist cultural and institutional structures and barriers, it is important to understand the unique experiences of sexual minority women from diverse racial/ethnic backgrounds. Parks et al. (2004) provide data from 448 lesbian women of diverse racial/ethnic backgrounds who participated in the Chicago Health and Life Experiences of Women study. These authors examined the intersections of race/ethnicity and sexual identity development by comparing African American, Latina, and White Lesbian women on a variety of identity-related measures. Their findings indicated that African American and Latina respondents differ little in terms of ages at which they reported sexual identity development milestones and levels of sexual identity disclosure. However, comparisons between women of color collectively and White women revealed significant variability. These findings highlight the importance of examining the extent to which existing models of sexual identity development are inclusive of the experiences of women from cultural minority backgrounds. 


\section{Summary}

Developmental frameworks that delineate the formation of a sexual minority identity must consider differential socialization experiences of women and men as well as fundamental differences in sexuality. Institutionalized sexism and gender socialization considerably influences how women perceive themselves individually and collectively (Holmes, 2001). Rather than broadly applying a developmental framework centered on the experiences of men to women, comparable attention must be given to the experiences of women in deriving an identity formation model.

\section{Models of Multiple Identity Development}

Models of sexual minority identity development sensitive to the complex nature of integrating several identities are considered in the following section. There are relatively few models that exist in this regard.

Morales (1990) provided an identity formation framework for gay and lesbian racial/ethnic minority persons. This framework represented one of the earlier approaches to understanding the experience of managing multiple minority identities. Five different stages are outlined in this model: denial of conflicts, bisexual versus gay/lesbian, conflicts of allegiances, establishing priorities in allegiances, and integrating the various communities. Each state facilitates decreased anxiety by managing the varying levels of conflict experienced by the individual. Other models of identity development, i.e., racial identity or sexual identity, also may influence how individuals work through issues in each state. Integration is achieved when the individual gains a better understanding of the self and is able to incorporate a multicultural perspective to his/her life. 
Myers, Speight, Highlen, Cox, Reynolds, Adams, and Hanley (1991) suggested that systems of societal oppression have served to marginalize individuals as a whole and to segregate pieces of an individual's identity. This eventually limits the individual from developing a holistic and integrated sense of self. In an attempt to provide a comprehensive framework, they argue for an optimal theory approach to identity development. Using this approach, identity development is viewed as a process that seeks to "integrate all apparent aspects of being (e.g., age, color, ethnicity, and size) into a holistic sense of self" (Myers et al., 1991, p.58). Thus, individuals may progress through seven stages of development to achieve an integrated identity: absence of conscious awareness, individuation, dissonance, immersion, internalization, integration, and transformation.

Reynolds and Pope (1991) offered a more particular multidimensional identity model as opposed to the general model of managing multiple aspects of identity posited by Myers et al. (1991). This perspective provides a detailed explanation of how and why individuals resolve competing issues relevant to multiple identities. Four possible options are suggested for identity resolution: identification with one aspect of self based on societal expectations, identification with one aspect of self based on personal expectations, identification with multiple aspects of self but presenting identity in segmented ways, and identification with a combined aspect of self through recognizing the intersection among multiple identities. Individuals may move from one option to another option to achieve an integrated identity depending on their personal needs, needs of a reference group with which they are affiliated, as well as the general environment in 
which they live. Each option for the individual is viewed as an acceptable option for an integrated identity because the development of positive self-esteem is feasible.

As with other identity development or formation models, the contribution to the literature made by Reynolds and Pope (1991) has been conceptualized theoretically and has yet to be tested empirically on the population to which it applies. Recognizing the lack of developmental models inclusive of the experiences of racial/ethnic minorities and sensitive to the divergent socialization experiences of women and men, Fassinger and colleagues conceptualized a model that exceeds the confines of earlier theories. The model departs from earlier stage models in recognizing that at single linear pathway is an inadequate at best in capturing the diverse experiences associated with sexual minority identities (Croteau et al., in press). This model is presented in the following section.

\section{Inclusive Model of Sexual Minority Identity Formation}

McCarn and Fassinger's (1996) model represents an attempt to address the limitations noted in existing models and is intended to be broadly inclusive of the diverse paths one may take to an integrated and synthesized lesbian or gay identity. The model is similar to other identity models in that the process of identity development is conceptualized in terms of phases as opposed to stages as it implies greater flexibility. Also, while the phases are outlined in a progression for the ease of description, the authors conceptualized the process as continuous and circular. As individuals encounter new relationships, issues regarding individual sexual identity may be renegotiated. Similarly, as individuals encounter new contexts, an awareness of group oppression is likely to be solidified. Thus, re-cycling and simultaneous location in multiple phases is possible and the two distinct processes potentially but not necessarily influence one 
another. In deriving the model, the authors draw from the racial/ethnic identity literature the concept of three relevant attitude areas at each phase of group identity development: attitudes toward self, toward other sexual minority persons and toward sexual majority persons. Items in each instrument (lesbian women; McCarn \& Fassinger, 1996; gay men, Fassinger \& Miller, 1996) also reflect considerations of gender differences in the sexual minority identity formation process. For example, based on understandings of gender identity and the effects of gender in the process of self-disclosure, items in the instrument for women reflect greater consideration of a relational or other-oriented approach to sexuality.

What distinguishes this model from other sexual minority identity development models is that self-disclosure of identity does not represent confirmation of developmental advancement. Self-disclosure of identity represents a single dimension of experience germane to the process of sexual minority identification (Mohr \& Fassinger, 2000) and has been conceptualized as an interpersonal variable that involves the revealing of one's sexual minority orientation and participation in sexual minority community activities. This is distinct from sexual minority identification as it represents an intrapersonal variable involving movement from ambivalence to internalization of sexual minority identity (Mohr \& Fassinger, 2000). Thus, non self-disclosure of sexual minority orientation is not necessarily indicative of negative identity. "Disclosure is so profoundly affected by environmental oppression that to consider it as an index of identity development directly forces an individual to take responsibility for her/his own victimization” (McCarn \& Fassinger, 1996, p. 522). 
The authors propose a four phase model with two parallel branches that separate the individual process from the group membership process. An initial phase of nonawareness precedes both branches of the model, and the four subsequent phases (awareness, exploration, deepening/commitment, internalization/synthesis) follow similarly in each branch. Thus, the proposed model for validation represents eight phases in the identity formation process (four phases of identity within each branch).

\section{Individual Sexual Identity Formation}

Phase 1: Awareness. The onset of a minority sexuality begins with awareness of feeling or being different. Sensations and desires that diverge from the heterosexual norm are likely to be experienced and attributed to the self. One begins to question assumptions of a heterosexual identity. Affective states experienced include confusion, fear, and/or bewilderment.

Phase 2: Exploration. This second phase involves one actively exploring strong (often erotic) feelings about other same-sex people or a particular same-sex person. Questions that arise from phase one are actively considered. Affective states experienced include longing, excitement, and wonder, as formerly unknown aspects of one's sexuality are discovered.

Phase 3: Deepening/commitment. The experience of phase two leads the individual to a deepening of sexual and emotional self-knowledge. In this phase, choices concerning sexuality become solidified. The individual recognizes that preferences for certain forms of intimacy imply a particular identity. This identity is then considered in the context of pervasive heterosexism and homonegativity. Affective states include anger, sadness as well as acceptance and self assurance. 
Phase 4: Internalization/Synthesis. In this phase, the individual fully internalizes same-sex desire/love as a part of overall identity. A sense of internal consistency is likely to be manifested; characterized by certainty regarding preferences and contentment and pride about those preferences.

\section{Group Membership Identity Formation}

Phase 1: Awareness. The individual enters this phase as he or she realizes that heterosexuality is not a universal norm and that diverse sexual orientations exist. The realization of a community of lesbian and gay persons may further create a consciousness of heterosexism.

Phase 2: Exploration. In this phase, the individual seeks to define her/his position in relation to the reference group along two dimensions: attitudes and membership. The individual in this phase begins to actively pursue knowledge about same-sex communities and considers belonging to such a community.

Phase 3: Deepening/commitment. A deepening awareness of both the unique value and oppression of the lesbian/gay community characterizes this phase. A commitment to create a personal relationship to the reference group is involved. Awareness of possible consequences of such commitment is manifested.

Phase 4: Internalization/Synthesis. The individual enters this final phase as she or he has moved through a process of conflict and reevaluation, identified as a member of a minority group, redefined the meaning of that group, internalized this new identity, and synthesized it into the overall self-concept. 
Scholars in the area of sexual minority identity formation have noted the model's inclusiveness (e.g., Firestein, 2007; Liddle, 2007; Potoczniak, 2007) and applicability to counseling practice (e.g., Bieschke, Paul, \& Blasko, 2007).

\section{Statement of the Problem}

Over the past three decades there has been significant growth in the psychological literature that affirmatively explores sexual minority identity (Croteau et al., 2007). Similarly, there has been a considerable increase in the literature on racial/ethnic identity and the relationship of race/ethnicity to other psychological variables (Fouad \& Brown, 2000). In both areas, there have been critical discussions regarding the effects of membership in institutionally oppressed groups on mental health and well-being. Although a rapidly expanding body of literature reflects exploration of each area from a wide range of perspectives, relatively little integrative attention is given to sexual minorities who also represent a racial/ethnic minority identity.

A majority of the empirical research that focuses on sexual minorities, particularly on lesbian women and gay men, is conducted with a White, middle-class sample (Parks et al., 2004). Further, research on members of racial/ethnic minority groups rarely acknowledges differences in sexual orientation of group members. Thus, there is little exploration of the complex intersection of sexual minority identity and racial/ethnic identity (Bieschke et al., in press). What is more, the realistic social tasks and stressors that are a component of sexual minority identity formation in conjunction with racial/ethnic identity formation are ignored. Such biases are rarely highlighted in articles or in statements explaining the limited generalizability of findings (Greene, 1994). Moreover, such narrow research perspectives result in a limited understanding of the 
diversity within these groups, particularly regarding the development of individual identity and affiliation to group identity (McCarn \& Fassinger, 1996).

Further, an understanding of the meaning of being a sexual minority person of color requires a careful exploration of the importance of cultural gender roles and of both the nature and relative fluidity or rigidity of a culture's traditional gender stereotypes (Alquijay, 1997; Smith, 1997; Chan, 1997; Greene, 2000). Moreover, sexual minority persons of color encounter unique challenges stemming from racism within the mainstream sexual minority community (Chung \& Katayama, 1996), conflicting cultural values (Chan, 1995), competing identity groups (Alquijay, 1997), and the need to negotiate identity across context (Zea et al., 2003).

Until recently, most identity theories and models generally have discussed and examined a specific social identity reflecting an assumption that group members are homogenous and lack multiple identities (Fukuyama \& Ferguson, 2000). Some researchers have attempted to examine multiple layers of identity of culturally diverse individuals in identity formation theories (e.g., McCarn \& Fassinger, 1996). However, most existing identity theories and models continue to focus on homogenous characteristics of group members. One of the primary limitations of recognizing only single identities is that individuals who embrace multiple identities are often invisible members within specific social reference groups (Fukuyama \& Ferguson). Further, implications for improved health and well-being derived from a positive and integrated identity remain unexamined for those negotiating multiple identities. The HIV/AIDS epidemic has brought some attention to the experiences of sexual minority men of color. However, such attention is markedly low given the overrepresentation of HIV/AIDS 
among this population (Wilton, Halkitis, English, \& Roberson, 2005). By comparison, the experiences of sexual minority women of color remain largely invisible in the empirical literature (Harper et al., 2004).

For the sexual minority individual experiencing a unique and complex identity, it is appropriate to assume that this identity will be influenced by the interrelation of several factors, including one's cultural (i.e., racial/ethnic) and gender orientation (Fassinger \& Arseneau, 2007). The measure undergoing evaluation in the current study is thus intended to adequately assess one's location in the sexual minority identity formation process while remaining sensitive to the influence of his/her current gender and cultural locations. Specifically, the current work assessed the reliability and validity of a sexual minority identity formation measure (the Same-Sex Orientation Identity Questionnaire derived from the McCarn \& Fassinger, 1996 model) with a racially/ethnically diverse population.

\section{Hypotheses}

The current study examined the following hypotheses:

Hypothesis 1: Internal consistency reliability: The eight subscales of the SameSex Orientation Identity Questionnaire (SSOIQ) will produce adequate estimates of internal consistency reliability for the current sample as measured by Cronbach's alpha.

Hypothesis 2: Structural validity: The theoretical factor structure of the SSOIQ will hold with a sample of racial/ethnic minority persons as assessed formally through factor analysis.

Hypothesis 3: Convergent validity: The eight subscales will correlate significantly in expected directions with the following constructs: (a) identity confusion, (b) internalized homonegativity, (c) same group orientation, and (d) outness. 


\section{Hypothesis 3a: Identity Confusion.}

1. Identity Confusion will be positively and significantly correlated with the two earlier phases of Individual Sexual Identity (Awareness and Exploration).

2. Identity Confusion will be negatively and significantly correlated with the two later phases of Individual Sexual Identity (Deepening/Commitment and Internalization/Synthesis).

Identity confusion reflects the first step toward integrating a sexual minority identity into the self-concept (Mohr \& Fassinger, 2000). This process involves the individual's questioning assumptions about one's sexual orientation and feelings of isolation and alienation from the heterosexual norm. Identity confusion is thus parallel to the initial phase of awareness and theoretically incongruent with the final step of internalization/synthesis. In this final phase, the sexual minority person experiences a firm self-acceptance of desire/love for members of the same gender as part of his or her overall identity. In the initial awareness phase, an individual becomes cognizant of feeling "different" from the heterosexual norm. Affective states in this phase are similar to those in the identity confusion phase and include confusion, fear, and bewilderment.

\section{Hypothesis 3b: Internalized Homonegativity.}

1. Internalized Homonegativity will be positively and significantly correlated with the two earlier phases of Individual Sexual and Group Membership Identity (Awareness and Exploration).

2. Internalized Homonegativity will be negatively and significantly correlated with the two later phases of Individual Sexual and Group Membership Identity (Deepening/Commitment and Internalization/Synthesis). 
Internalized homonegativity is conceptualized as the psychological consequence of living in a homonegative and heterosexist society (Bohan, 1996). This construct refers to a hatred or condemnation of same-sex sexuality that has been internalized by sexual minority persons. Items evaluating representations in the final phases of the identity process (synthesis of individual and group identity) assess feelings of self-acceptance, pride, comfort, and security regarding one's sexual minority identity. Such affective states are inconsistent with the self-denigration implied in internalized homonegativity.

\section{Hypothesis 3c: Same Group Orientation.}

1. Same Group Orientation will be negatively and significantly correlated with the two earlier phases of Group Membership Identity (Awareness and Exploration).

2. Same Group Orientation will be positively and significantly correlated with the two later phases of Group Membership Identity (Deepening/Commitment and Internalization/Synthesis).

Same group orientation is conceptualized as the extent to which one self-identifies with his/her social group, engages in behaviors and practices specific to his/her particular social group, and has a sense of affirmation and belonging to his/her social category (Phinney, 1992). Group synthesis of identity as conceptualized in the current model represents a firmly internalized identity as a member of an oppressed group into the overall self-concept. A synthesized group identity is characterized by feelings of comfort, fulfillment, security, and an ability to maintain one's sense of self as a sexual minority person across context. It is likely that some self-disclosure has occurred in this phase. Group awareness, however, is characterized by a new consciousness of the various sexual orientations individuals may represent. Also, the individual in group awareness is often 
forced to acknowledge that heterosexism exists. Thus, participants endorsing items that indicate internalization/synthesis of sexual minority group identity will similarly endorse items on the measure of same group orientation that reflect identification and interaction with sexual minority communities. The opposite will be true for sexual minority group identity awareness.

\section{Hypothesis 3d: Outness}

1. Outness will be negatively and significantly correlated with the two earlier phases of Group Membership Identity (Awareness and Exploration).

2. Outness will be positively and significantly correlated with the two later phases of Group Membership Identity (Deepening/Commitment and Internalization/Synthesis).

Self-disclosure of identity represents a single dimension of experience connected to the process of sexual minority identification (Mohr \& Fassinger, 2000). Self-disclosure has been conceptualized as an interpersonal variable that involves the revealing of one's sexual minority orientation and participation in sexual minority community activities. This is distinct from sexual minority identification as it represents an intrapersonal variable subsuming internalized homonegativity and confusion about one's sexual orientation identity (Mohr \& Fassinger). Thus, non self-disclosure of sexual minority orientation is not necessarily indicative of negative individual identity. However, the process of integrating a positive and affirmed identity as a member of an oppressed social group implies some community involvement. The degree to which one has self-disclosed can be assessed in different spheres of life; for example family, friends, work, religious institutions, and to the general public (Mohr \& Fassinger). Further, level of outness in one sphere of functioning may be somewhat related to those in another sphere of 
functioning, yet outness in all spheres aggregated together represent one's general level of outness.

Hypothesis 4: Discriminant validity: The eight subscales will not correlate significantly with dogmatism.

Dogmatism refers to a relatively closed cognitive structure of beliefs about reality, organized around a central set of beliefs regarding absolute authority (Rokeach, 1956). This closed cognitive structure, in turn, provides a framework for patterns of intolerance and qualified tolerance toward others. Thus, the construct of dogmatism subsumes three variables; closed cognitive systems, general authoritarianism, and general intolerance. The proposed instrument intends to measure one's phase in a minority identity formation process. While items assess the content of one's beliefs to the extent that they are affirming of a stigmatized identity, they do not evaluate the structure of the individual's belief system, that is, the extent to which one rigidly adheres to his/her liberal and affirming beliefs. Because the model intended for validation is characteristically fluid and dynamic, we expect that those who "successfully" move through the process would exhibit some degree of cognitive flexibility. However, we conceptualize the process of identity formation as distinct from the construct of cognitive flexibility; thus, we expect a small and non-significant correlation. 


\section{Chapter 3: Method}

\section{Design Statement}

The current study used a descriptive field design in which correlational analyses were conducted. Descriptive field studies are characterized by high external and low internal validity. The proposed study did not exercise experimental control (randomization, manipulation of variables) and was conducted in a real world setting; participants were recruited directly from the population of interest (sexual minority persons of color). The variables of interest were assessed through self-report measures. Reliability and validity of the proposed measure was assessed through correlational analyses.

\section{Item Development}

There were two phases of the item development process. The first phase involved refining items from two previously developed measures (women and men's versions) with the input from a research team. The second phase involved piloting the items with individuals who represent the population of interest. The steps undertaken in phase one and two are described in greater detail below.

Phase one: Item revision. A research team of nine graduate students conducting sexual minority research and conversant with the work of Fassinger and colleagues (McCarn \& Fassinger, 1996; Fassinger \& Miller, 1996) were involved in the item revision process. Team members were diverse in terms of sexual orientation, gender, and graduate program. Of the known sexual orientations, there were four gay men, one bisexual woman, one lesbian woman, and two heterosexual women. One team member was in a doctoral program for American studies, another was in a masters program for 
college student personnel, and the remaining team members were in a doctoral program for counseling psychology or a counseling related field. In terms of race/ethnicity, one team member identified as Latina and the remainder identified as White.

Members of the team evaluated a pool of previously developed items (see Fassinger, 2001a, 2001b) using an item revision worksheet developed by the authors (see Appendix A). Members of the team were asked to code each item as reflecting one or more of the following; cognition, emotion, behavior, knowledge, relational, or cultural context. Members were also asked to determine if each item should be retained as is, eliminated, or revised. Team members were then asked to comment on the items and suggest revisions. The feedback from the team was used in developing the final pool of revised items.

In regards to changes made to the original items, more inclusive language was used to reference sexual minority communities (e.g., "gay" was replaced with "gay, bisexual, queer" in the men's survey). The readability of certain items was made more accessible to community populations (e.g., "Now that I am consistently doing what I want to do in terms of love and sex, I feel more integrated as a person" was changed to "I feel more complete as a person because I am consistently doing what I want to do in terms of love and sex"). The directions of the original measure were condensed. Finally, temporal language (e.g., "recently" or "lately") was removed from the individual items and emphasized in the directions.

Phase two: Piloting of items. Ten sexual and racial/ethnic minority persons (six men and four women) reviewed the statements using a card-sort technique. The women ranged in age from 18 to 33 years. Two self-identified as lesbian and two self-identified 
as queer. One woman identified as Asian American and the remaining three women identified as African American. The men ranged in age from 17 to 27 years. Five men self-identified as gay and one self-identified as queer. Three men identified as African American, two men as Hispanic/Latino/Chicano, and one man as Middle Eastern.

The items were written on individual index cards and shuffled. Cards were then presented to each participant who was instructed to sort the cards into two equally sized groups of statements representing individual identity processes and group membership identity processes. Next, participants were asked to sort the items within each of the two groups into four equally sized piles. Finally, participants were asked to place the four piles within each of the two groups into sequential order. Participants were then asked to identify where they see themselves in the sexual minority identity formation process. Card sort instructions and participant handouts can be seen in Appendices B and C respectively.

Frequencies for correct item placement were tabulated and patterns of misplaced items were determined. The frequencies for correct item placement were adequate given the small sample size. An item was considered problematic if one person misplaced it in the women's group (cut off of 75\%) and two people misplaced it in the men's group (cut off of $66.7 \%)$. Based on the number of problematic items per phase, women card sort participants had some difficulty differentiating between the Group Exploration phase and the Group Deepening/Commitment phase. Men card sort participants had some difficulty differentiating between the Group Exploration, Group Deepening/Commitment, and Individual Deepening/Commitment phases. Overall, results of the card sort did not indicate major problems with the categorization of the items and were consistent with 
previous research (Fassinger \& Miller, 1996) that obtained clearer results for the early and late phases when compared to the middle phases. Minor revisions were made to the items (e.g., "homophobic" was added next to the word "homonegative" on an item for men) based on feedback from card-sort participants prior to proceeding to the online study.

\section{Participants}

Participants were 98 sexual and racial/ethnic minority persons. Ten participants were dropped from the final sample of participants for leaving more than $70 \%$ of the survey blank. Also, 19 participants were dropped for not indicating a gender and/or indicating transgender for their primary gender identification. Thus, out of the 98 participants, data from 69 participants (37 men, 32 women) were analyzed in the current study. The final sample size obtained provided sufficient power to conduct correlations. Results of a conservative power analysis (power $=.80, \mathrm{~d}=.70$, alpha $=.05$ ) yielded a minimum of 33 participants per correlation (Cohen, 1988).

For the women's sample $(\mathrm{N}=32)$, participants ranged in age from 18 to 51 years $(M=26.44 ; S D=7.47 ; M d n=24.50)$. Approximately $37.5 \%$ of participants were undergraduate students, $28.1 \%$ were graduate students, and $21.9 \%$ were not students (12.5\% responded other). In terms of annual household income, $40.6 \%$ of participants reported low income (less than 24,999), 34.3\% middle income (between 25,000 and 149,999), 9.4\% high income (greater than 150,000), and $15.6 \%$ indicated "don't know." Race/ethnicity was assessed through self-report and participants were able to select multiple racial/ethnic identifications. Fifty percent identified as African American, 21.9\% as Asian American, $15.6 \%$ as Hispanic/Latina/o, $15.6 \%$ as White, $9.4 \%$ as Native 
American, 6.3\% as Middle Eastern, 3.1\% as Multiracial, 3.1\% as South East Asian, and 9.4\% indicated "other" for race/ethnicity. All participants indicated that they identified as a "person of color" and/or "racial/ethnic minority." Participants reported having lived in the U.S. from 10 years to 51 years. Participants' self-reported sexual orientations were diverse. Participants were able to select multiple sexual minority identifications. Fifty percent identified as lesbian, $40.6 \%$ queer, $34.4 \%$ bisexual, $18.8 \%$ gay, $18.8 \%$ same-sex oriented or attracted, $12.5 \%$ questioning, and $15.6 \%$ as "other" for sexual orientation.

For the men's sample $(\mathrm{N}=37)$, participants ranged in age from 19 to 35 years $(M$ $=24.78 ; S D=4.16 ; M d n=25)$. Approximately $32.4 \%$ of participants were not students, $29.7 \%$ were undergraduate students, and $29.7 \%$ were graduate students $(2.7 \%$ responded other). In terms of annual household income, $64.8 \%$ of participants reported middle income (between 25,000 and 149,999), 21.6\% low income (less than 24,999), 2.7\% high income (greater than 150,000), and 10.8\% indicated “don't know." Race/ethnicity was assessed through self-report and participants were able to select multiple racial/ethnic identifications. Thirty-five percent identified as Hispanic/Latina/o, 32.4\% as Asian American, 13.5\% African American, $13.5 \%$ as South East Asian, 10.8\% as Multiracial, 5.4\% as Native American, 5.4\% as White, $0 \%$ as Middle Eastern, and 0\% indicated “other" for race/ethnicity. All participants indicated that they identified as a "person of color" and/or "racial/ethnic minority." Participants reported having lived in the U.S. from 10 years to 31 years. Participants' self-reported sexual orientations were diverse. Participants were able to select multiple sexual minority identifications. Eighty percent identified as gay, $54.1 \%$ queer, $16.2 \%$ bisexual, $13.5 \%$ same-sex oriented or attracted, $10.8 \%$ questioning, and $2.7 \%$ as "other" for sexual orientation. 


\section{Measures}

Sexual minority identity formation. The Same-Sex Orientation Identity

Questionnaire (SSOIQ; 2007) is a 40 item self-report, Likert-type scale that assesses dimensions of sexual identity formation along two branches: Individual Sexual Identity and Group Membership Identity. There is a women's version and men's version of the measure. Items are rated on a 7-point scale ranging from disagree strongly (1) to agree strongly (7). There are items pertaining to each of four phases of identity development (Awareness, Exploration, Deepening/Commitment, and Internalization/Synthesis) within each branch. Sample items reflecting Internalization/Synthesis include "I feel more complete as a person because I am consistently doing what I want to do in terms of love and sex" (Individual Sexual Identity, women and men's version) and "My sexuality is an integrated part of my social and public life" (Group Membership Identity, women and men's version). Sample items reflecting Awareness include "It scares me that I am not exclusively attracted to women" (Individual Sexual Identity, men's version) or "I wonder what it might be like to be romantic with a woman" (Individual Sexual Identity, women's version) and "There may be men out there who have the same kinds of sexual desires that I do" (Group Membership Identity, men's version) or “There may be women out there who have the same kinds of sexual desires that I do" (Group Membership Identity, women's version).

There are two approaches to scoring the SSOIQ. In the first approach, each respondent receives a separate mean score for each of the eight subscales that represent the phases of the model. In the second approach, respondents can be categorized into a predominant phase for each of the two branches by first obtaining a mean score for each 
phase and then identifying the largest mean score for the Individual and Group branches as the predominant phase. In the current study, mean scores on all eight subscales were used, as identifying only one predominant phase location was not relevant to the objective of the study. Preliminary evidence of the scales' validity has been supported in samples of lesbian women (Fassinger \& McCarn, 1991; McCarn, 1991) and gay men (Fassinger \& Miller, 1996) using a Q-sort methodology. Although these studies had small samples, participants were somewhat diverse in terms of age and cultural demographics.

\section{Identity confusion and internalized homonegativity. The Lesbian and Gay Identity}

Scale (LGIS; Mohr \& Fassinger, 2000) is a 40-item self-report, Likert-type scale that assesses a wide range of feelings and beliefs related to sexual minority identity. Subscales that reflect negative feelings and beliefs related to one's sexual orientation include Internalized Homonegativity (5 items) and Identity Confusion (4 items). For the purposes of the current study, only items from the Internalized Homonegativity and Identity Confusion subscales were included in the survey. Items are rated on a 7-point scale ranging from disagree strongly (1) to agree strongly (7). Sample items from the Internalized Homonegativity subscale include "I would rather be straight if I could" and "I am glad to be a LGBQ person." Sample items from the Identity Confusion subscale include "I keep changing my mind about my sexual orientation" and "I am not totally sure that I'm a LGBQ person."

Mohr \& Fassinger (2000) reported a full sample alpha of .79 (.78 for lesbians and .79 for gay men) for the Internalized Homonegativity subscale and .77 (.79 for lesbians and .65 for gay men) for the Identity Confusion subscale. In regard to validity, scores on the Internalized Homonegativity subscale demonstrated negative and significant 
correlations with self-esteem, same group orientation, and involvement in religious communities for gay men participants (Mohr \& Fassinger). For lesbian women participants, scores on Internalized Homonegativity demonstrated negative and significant correlations with same group orientation. Further, scores representing Internalized Homonegativity demonstrated negative and significant correlations with internalization/synthesis. Validity of the Identity Confusion subscale was demonstrated by negative and significant correlations with self-esteem, same group orientation, and internalization/synthesis for lesbian women participants.

Same group orientation. The Same Group Orientation (SGO) scale of the Multigroup Ethnic Identity Measure (MEIM; Phinney, 1992) is a 14-item self-report, Likert-type scale that assesses positive cultural attitudes and sense of belonging (Affirmation/Belonging, 5 items), cultural identity achievement including both exploration and resolution of identity issues (Achievement, 7 items), and cultural behaviors or practices (Behavior, 2 items). Items are rated on a 4-point scale ranging from disagree strongly (1) to agree strongly (4). The instrument was designed for use with members of minority groups to measure the degree of same group orientation; items are worded to reflect the specific minority group that is being studied. Mohr and Fassinger's (2000) modified version of the SGO scale for use with sexual minority cultural groups was used in the current study. A sample item includes "I have spent time trying to find out more about the LGBQ community." Participants were instructed to reflect on their particular sexual minority group in responding to the items; items were modified accordingly. 
Mohr \& Fassinger (2000) reported an internal consistency estimate of .88 with a sample of lesbian women and gay men. In regard to validity, scores on the SGO demonstrated a positive and significant correlation with self-esteem among racial/ethnic minority high school and college students (Phinney, 1992). Further, scores on the modified version of the SGO for use with sexual minority communities have demonstrated positive and significant correlations with outness and negative and significant correlations with internalized homonegativity and identity confusion (Mohr \& Fassinger).

Outness. The Outness Inventory (OI; Mohr \& Fassinger, 2000) is a 10-item selfreport, Likert-type scale that measures the degree to which a respondent's sexual orientation is known by and openly discussed with individuals in different spheres of the person's life (i.e., degree to which a respondent is "out" about her or his sexual orientation). Each item consists of a particular individual (i.e., mother) or type of individual (e.g., work peers) that is rated by using a fully anchored 7-point scale ranging from (1) person definitely does not know about your sexual orientation status to (7) person definitely knows about your sexual orientation status, and it is openly talked about. The OI consists of three subscales; Out to World, Out to Family, Out to Religion. Mohr \& Fassinger reported an alpha of .79 for the Out to World subscale (4 items), .74 for the Out to Family subscale (4 items), and .97 for the Out to Religion subscale (2 items). In regard to validity, scores on the OI correlated significantly as predicted with same group orientation for both lesbian women and gay men.

Dogmatism. The short form of Rokeach's Dogmatism Scale (RDS; Troldahl \& Powell, 1965) is a 20-item self-report, Likert-type scale that assesses the respondent's 
style of belief systems. Respondents were instructed to determine the extent to which they agree or disagree with a statement. Items were rated on a 7-point scale ranging from disagree strongly (1) to agree strongly (6). Sample items include "My blood boils whenever a person stubbornly refuses to admit he's wrong" and "It is better to be a dead hero than to be a live coward." Items were modified to be more gender inclusive (e.g., "he" changed to "he/she"). Troldahl \& Powell (1965) reported a lower limit reliability of .73 and a split-half reliability of .79 for the short form of the RDS. In regard to validity, scores on the short form of the RDS demonstrated a negative and significant correlation with attitudes toward feminism and the women's movement (Fassinger, 1994).

\section{Procedure}

Participants were recruited from student and community organizations, sexual minority affirmative churches, online social networking websites, and settings/businesses that target sexual minority communities of color. Participants also were recruited through personal contacts of the authors and snowball sampling.

Advertisements for the study were sent through electronic mail (e-mail) listservs, posted at recruitment locations, and printed in local sexual minority newsletters. Email and snowball sampling efforts resulted in a participant pool from a wider geographic area. With this in mind, items on the demographic questionnaire were included to track the participants' geographic region and from where they received information about the study.

Further, announcements for the study were made in person at organization/group meetings and events. In-person recruitment efforts were made in light of recommendations for sampling sexual minority communities of color. Croom (1999) 
suggests that racial/ethnic minority persons report feeling that their unique experiences are not valued or are overlooked when participating in research. Racial/ethnic minority persons also report feeling distrustful of White researchers (Croom). Thus, culturally sensitive efforts that communicate appreciation and regard for participation are recommended to recruit individuals from underrepresented populations.

Recruitment material described eligibility criteria and stated the authors' interest in the life experiences of people of color with same-sex attractions (see Appendix D). This material provided the web address for the online survey. Time to complete the survey was approximated at 20 to 30 minutes. As an incentive, participants were given the option to enter a drawing for one of two $\$ 50$ gift cards upon submitting the survey. All participants received written informed consent and debriefing. These materials explained eligibility requirements, the purpose of the study, procedures, confidentiality, risks, benefits, freedom to withdraw, and provided contact information for the researchers, the IRB office, and national LGBT resources. See Appendices E, F, and G for informed consent, survey, and debriefing (respectively). 


\section{Chapter 4: Results}

The SSOIQ was developed to measure one's location in a sexual minority identity formation process. Each hypothesis was tested on the women and men's samples separately. Hypothesis 1 tested the internal consistency reliability for the current sample for each of the eight subscales. Hypothesis 2 intended to test the structural validity through a confirmatory factor analysis. However, because the minimum sample size required for a confirmatory factor analysis was not obtained, an examination of the intercorrelations of the eight subscales was conducted as a preliminary evaluation of the theoretical model. Hypotheses 3-4 examined the psychometric properties of the SSOIQ; specifically, Hypotheses 3a-3d tested convergent validity and Hypothesis 4 tested discriminant validity.

Table 1 contains the means and standard deviations of scores for all measures for the sample of women. As can be seen in Table 1, the possible range of scores for each of the eight subscales of the SSOIQ was 1-7. The mean and standard deviation for each of the eight subscales were as follows; Individual Awareness $(M=4.6, S D=1.2)$,

Individual Exploration $(M=6.0, S D=.92)$, Individual Deepening/Commitment $(M=6.1$, $S D=.94)$, Individual Internalization/Synthesis $(M=5.6, S D=1.2)$, Group Awareness $(M$ $=4.6, S D=.81)$, Group Exploration $(M=4.8, S D=.75)$, Group Deepening/Commitment $(M=4.0, S D=.97)$, Group Internalization Synthesis $(M=5.6, S D=.87)$.

In regards to the convergent and discriminant validity measures, for the sample of women the mean score on the Identity Confusion scale was 2.4 with a standard deviation of 1.6. The mean score on the Internalized Homonegativity scale was 2.2 with a standard deviation of 1.3. The mean score on the Same Group Orientation scale was 3.8 with a 
standard deviation of .64. The mean score on the Outness scale was 4.7 , with a standard deviation of 1.5. Finally, the mean score on the Dogmatism scale was 2.9 with a standard deviation of .70. Alphas for the convergent and discriminant validity measures for the sample of women ranged from .79 to .91 and can be seen in Table 1 .

Table 1

Scale Ranges, Means, and Standard Deviations for Women

\begin{tabular}{lcccc}
\hline \multicolumn{1}{c}{ Scale } & Possible Range & Mean & SD & $\propto$ \\
\hline Individual Awareness & $1-7$ & 4.6 & 1.2 & - \\
Individual Exploration & $1-7$ & 6.0 & .92 & - \\
Individual Deepening/Commitment & $1-7$ & 6.1 & .94 & - \\
Individual Internalization/Synthesis & $1-7$ & 5.6 & 1.2 & - \\
Group Awareness & $1-7$ & 4.6 & .81 & - \\
Group Exploration & $1-7$ & 4.8 & .75 & - \\
Group Deepening/Commitment & $1-7$ & 4.0 & .97 & - \\
Group Internalization/Synthesis & $1-7$ & 5.6 & .87 & - \\
Identity Confusion & $1-7$ & 2.4 & 1.6 & .79 \\
Internalized Homonegativity & $1-7$ & 2.2 & 1.3 & .85 \\
Same Group Orientation & $1-4$ & 3.8 & .64 & .91 \\
Outness & $1-7$ & 4.7 & 1.5 & .88 \\
Dogmatism & $1-6$ & 2.9 & .70 & .82 \\
\hline
\end{tabular}

Table 2 contains the means and standard deviations of scores for all measures for the sample of men. As can be seen in Table 2, the possible range of scores for each of the eight subscales of the SSOIQ was 1-7. The mean and standard deviation for each of the eight subscales were as follows; Individual Awareness $(M=3.9, S D=.79)$, Individual Exploration $(M=5.5, S D=.67)$, Individual Deepening/Commitment $(M=6.1, S D=.82)$, Individual Internalization/Synthesis $(M=5.5, S D=1.1)$, Group Awareness $(M=3.4, S D$ $=.95)$, Group Exploration $(M=4.5, S D=1.1)$, Group Deepening/Commitment $(M=4.9$, $S D=1.0)$, Group Internalization Synthesis $(M=5.4, S D=.94)$.

In regards to the convergent and discriminant validity measures, for the sample of men the mean score on the Identity Confusion scale was 1.7 with a standard deviation of 
1.1. The mean score on the Internalized Homonegativity scale was 2.3 with a standard deviation of 1.3. The mean score on the Same Group Orientation scale was 3.7 with a standard deviation of .70. The mean score on the Outness scale was 4.3, with a standard deviation of 1.3. Finally, the mean score on the Dogmatism scale was 3.0 with a standard deviation of .70. Alphas for the convergent and discriminant validity measures for the sample of men ranged from .81 to .91 and can be seen in Table 2 .

Table 2

Scale Ranges, Means, and Standard Deviations for Men

\begin{tabular}{lcccc}
\hline \multicolumn{1}{c}{ Scale } & Possible Range & Mean & SD & $\propto$ \\
\hline Individual Awareness & $1-7$ & 3.9 & .79 & - \\
Individual Exploration & $1-7$ & 5.5 & .67 & - \\
Individual Deepening/Commitment & $1-7$ & 6.1 & .82 & - \\
Individual Internalization/Synthesis & $1-7$ & 5.5 & 1.1 & - \\
Group Awareness & $1-7$ & 3.4 & .95 & - \\
Group Exploration & $1-7$ & 4.5 & 1.1 & - \\
Group Deepening/Commitment & $1-7$ & 4.9 & 1.0 & - \\
Group Internalization/Synthesis & $1-7$ & 5.4 & .94 & - \\
Identity Confusion & $1-7$ & 1.7 & 1.1 & .81 \\
Internalized Homonegativity & $1-7$ & 2.3 & 1.3 & .83 \\
Same Group Orientation & $1-4$ & 3.7 & .70 & .91 \\
Outness & $1-7$ & 4.3 & 1.3 & .84 \\
Dogmatism & $1-6$ & 3.0 & .70 & .83 \\
\hline
\end{tabular}

Hypothesis 1: Internal consistency reliability: The eight subscales of the SSOIQ will produce adequate estimates of internal consistency reliability for the current sample as measured by Cronbach's alpha. Cronbach's coefficient alpha represents the proportion of total variance in a given scale that can be attributed to a common source (DeVellis, 1991). The alpha coefficients for each of the eight subscales for both women and men are contained in Table 3. 
Table 3

Cronbach's Alpha for each subscale of the SSOIQ

\begin{tabular}{lcc}
\hline \multicolumn{1}{c}{ Scale } & $\propto$ SSOIQ Women & $\propto$ SSOIQ Men \\
\hline Individual Awareness & .63 & .26 \\
Individual Exploration & .73 & .37 \\
Individual Deepening/Commitment & .52 & .61 \\
Individual Internalization/Synthesis & .75 & .71 \\
Group Awareness & .34 & .63 \\
Group Exploration & .14 & .50 \\
Group Deepening/Commitment & .43 & .68 \\
Group Internalization/Synthesis & .52 & .52 \\
Individual Full Scale & .77 & .68 \\
Group Full Scale & .38 & .65 \\
\hline
\end{tabular}

The reliability coefficients ranged from .14 to .77 for women and .26 to .71 for men. The coefficients obtained for the Group Awareness (alpha = .34) and Group Exploration (alpha $=.14$ ) subscales for women demonstrate a poor level of reliability. Similarly, the coefficients obtained for the Individual Awareness (alpha $=.26)$ and Individual Exploration (alpha $=.37$ ) subscales for men demonstrate a poor level of reliability. The coefficients obtained for the Individual Awareness (alpha $=.63$ ), Individual Deepening/Commitment (alpha $=.52)$, Group Deepening/Commitment (alpha $=.43$ ), and Group Internalization/Synthesis (alpha $=.52)$ subscales for women demonstrate a weak level of reliability. Moreover, the coefficients obtained for the Individual Deepening/Commitment (alpha = .61), Group Awareness (alpha = .63), Group Exploration $($ alpha $=.50)$, Group Deepening/Commitment $($ alpha $=.68)$, and Group Internalization/Synthesis (alpha $=.52)$ subscales for men demonstrate a weak level of reliability. Finally, for women an adequate level of reliability was obtained for the Individual Exploration $($ alpha $=.73)$ and Individual Internalization/Synthesis $($ alpha $=$ .75) subscales. For men, an adequate level of reliability was obtained for the Individual 
Internalization/Synthesis $($ alpha $=.71)$ subscale. Thus, Hypothesis 1 was partially supported in that two of the eight subscales (i.e., Individual Exploration and Individual Internalization/Synthesis) produced an adequate estimate of internal consistency reliability for the sample of women and one of the eight subscales (i.e., Individual Internalization/Synthesis) produced an adequate level of internal consistency reliability for the sample of men.

It is worthy of note that the size of coefficient alpha is greatly influenced by the number of items in a given scale in proportion to sample size (Pett, Lackey, \& Sullivan, 2003). Given that each subscale only contained five items combined with the small samples $($ women $=32$, men $=37)$, these results should be interpreted with caution. To test if increasing the number of items would influence the alpha level, the alpha for the full scale Individual and Group branches (20 items each) were determined. The Individual full scale alpha coefficient for the sample of women demonstrated an adequate level of reliability (alpha $=.77$ ). For the sample of men, the Individual and Group full scale alphas approached and adequate level of reliability (.68 and .65 respectively).

Hypothesis 2: Structural validity: The theoretical factor structure of the SSOIQ will hold with a sample of racial/ethnic minority persons as assessed formally through factor analysis.

This hypothesis was unable to be tested at this point in time due to the small sample size. However, a preliminary examination of the intercorrelations among the eight subscales was conducted as a prelude to a formal confirmatory factor analysis. Kahn (2006) indicates the necessary sample size for a confirmatory factor analysis is well over 100 cases. 
In terms of expectations pertaining to the preliminary evaluation of the relationships between the subscales, a negative and significant correlation between the Awareness and Internalization/Synthesis subscales within each of the two branches was expected. Also, a positive and significant relationship between the four parallel phases was expected.

Alpha was set at .05 for all analyses. This decision was guided by a greater concern over a Type II error (not finding a true relationship) than a Type I error (incorrectly concluding that a true relationship exists). Table 4 contains the intercorrelations among all the variables of interest for women. As indicated in Table 4, the correlation between the Individual Awareness subscale and the Group Awareness subscale was .59 and significant at the .01 level. The correlation between the Individual Internalization/Synthesis subscale and the Group Internalization/Synthesis subscale was .65 and significant at the .01 level. There was no significant correlation between the Exploration or Deepening/Commitment subscales for the Individual and Group branches.

Finally, there was no significant correlation between the Awareness and

Internalization/Synthesis subscales for both the Individual and Group branches.

Table 4

Intercorrelations among Variables of Interest for Women

\begin{tabular}{|c|c|c|c|c|c|c|c|c|}
\hline Variable & 1 & 2 & 3 & 4 & 5 & 6 & 7 & 8 \\
\hline 1. Individual Awareness & 1 & & & & & & & \\
\hline 2. Individual Exploration & $.51 * *$ & 1 & & & & & & \\
\hline 3. Individual Deepening & .25 & $.66^{* *}$ & 1 & & & & & \\
\hline 4. Individual Internalization & -.25 & .30 & $.47 * *$ & 1 & & & & \\
\hline 5. Group Awareness & $.59 * *$ & .22 & -.11 & -.25 & 1 & & & \\
\hline 6. Group Exploration & .03 & .21 & .06 & 13 & .23 & 1 & & \\
\hline 7. Group Deepening & $.50 * *$ & $.45^{*}$ & .29 & .06 & .26 & .36 & 1 & \\
\hline 8. Group Internalization & -.10 & .33 & $.55^{* *}$ & $.65^{* *}$ & -.28 & .02 & .01 & 1 \\
\hline 9. Identity Confusion & .29 & -.15 & $-.52 * *$ & $-.53 * *$ & $.42 *$ & -.11 & -.24 & $-.36^{*}$ \\
\hline 10. Internalized Homonegativity & .35 & -.11 & -.33 & $-.50 * *$ & $.52 * *$ & .09 & -.04 &  \\
\hline 11. Same Group Orientation & -.08 & $.41 *$ & $.47 *$ & $.70 * *$ & $-.40 *$ & .23 & $.41 *$ & $.68 * *$ \\
\hline 12. Outness & $-.37 *$ & -.03 & .05 & $.42 *$ & $-.56 * *$ & -.13 & -.06 & $.42 *$ \\
\hline 13. Dogmatism & $.46^{*}$ & -.06 & .18 & -.00 & .30 & -.00 & .21 & -.23 \\
\hline
\end{tabular}


Table 5 contains the intercorrelations among all the variables of interest for men. As indicated in Table 5, the correlation between the Individual Awareness subscale and the Group Awareness subscale was .44 and significant at the .05 level. The correlation between the Individual Exploration subscale and the Group Exploration subscale was .47 and significant at the .01 level. The correlation between the Individual Internalization/Synthesis subscale and the Group Internalization/Synthesis subscale was .66 and significant at the .01 level. Also, there was a significant correlation between the Group Awareness subscale and the Group Internalization/Synthesis subscale $(r=-.45, p$ $<.01)$. There was no significant correlation between the Deepening/Commitment subscales for the Individual and Group branches. Finally, there was no significant correlation between the Awareness and Internalization/Synthesis subscales for the Individual branch.

Table 5

Intercorrelations among Variables of Interest for Men

\begin{tabular}{|c|c|c|c|c|c|c|c|c|}
\hline Variable & 1 & 2 & 3 & 4 & 5 & 6 & 7 & 8 \\
\hline 1. Individual Awareness & 1 & & & & & & & \\
\hline 2. Individual Exploration & .14 & 1 & & & & & & \\
\hline 3. Individual Deepening & -.32 & $.37 *$ & 1 & & & & & \\
\hline 4. Individual Internalization & -.25 & $.43^{*}$ & $.73 * *$ & 1 & & & & \\
\hline 5. Group Awareness & $.44 *$ & .05 & $-.39 *$ & $-.42 *$ & 1 & & & \\
\hline 6. Group Exploration & $.38 *$ & $.47 * *$ & .00 & -.01 & $.57 * *$ & 1 & & \\
\hline 7. Group Deepening & .33 & $.49 * *$ & .16 & .19 & .27 & $.57 *$ & 1 & \\
\hline 8. Group Internalization & -.16 & .26 & $.52 * *$ & $.66 * *$ & $-.45 * *$ & -.21 & -.11 & 1 \\
\hline 9. Identity Confusion & .22 & $-.37 *$ & $-.44 * *$ & $-.51 * *$ & $.48 * *$ & .13 & .05 & $-.60 * *$ \\
\hline 10. Internalized Homonegativity & $.55^{* *}$ & -.09 & $-.52 * *$ & $-.64 * *$ & $.69 * *$ & $.37 *$ & .08 & $-.54 * *$ \\
\hline 11. Same Group Orientation & -.27 & $.50 * *$ & $.58 * *$ & $.70 * *$ & $-.45^{*}$ & -.09 & .16 & $.55 * *$ \\
\hline 12. Outness & -.24 & .27 & .23 & .36 & -.37 & -.17 & .05 & .31 \\
\hline 13. Dogmatism & $.52 * *$ & .21 & -.10 & -.10 & .13 & .23 & $.53 * *$ & -.11 \\
\hline
\end{tabular}
Note. ${ }^{*} \mathrm{p}<.05,{ }^{*} \mathrm{p}<.01$.

Thus, the preliminary evaluation of the measure based on the intercorrelations between the subscales provided partial support for the theoretical model. That is, a negative and significant correlation between the Awareness and Internalization/Synthesis 
subscales in the group branch was found for men. Also, a positive and significant correlation was found between three of the four parallel phases (i.e., Awareness, Exploration, and Internalization/Synthesis). For the women's measure, support for the model was somewhat weak relative to that of the men's measure. A positive and significant correlation was found between the parallel Awareness and Internalization/Synthesis phases. The remaining expected relationships did not reach significance.

Hypothesis 3: Convergent validity: The eight subscales will correlate significantly in expected directions with the following constructs: (a) identity confusion, (b) internalized homonegativity, (c) same group orientation, and (d) outness.

As can be seen in Table 4, some of the correlations between the eight subscales of the SSOIQ and the measures of convergent validity were statistically significant in the predicted directions for women. As shown in Table 5, some of the correlations between the eight subscales of the SSOIQ and the measures of convergent validity were statistically significant in the predicted directions for men.

\section{Hypothesis 3a: Identity Confusion.}

1. Identity Confusion will be positively and significantly correlated with the two earlier phases of Individual Sexual Identity (Awareness and Exploration).

2. Identity Confusion will be negatively and significantly correlated with the two later phases of Individual Sexual Identity (Deepening/Commitment and Internalization/Synthesis).

For women, there was a negative and significant correlation between Identity Confusion and the two later phases of Individual Sexual Identity. The correlation 
between the Individual Deepening/Commitment subscale and Identity Confusion was $.52(p<.01)$ and the correlation between the Individual Internalization/Synthesis subscale and Identity Confusion was $-.53(p<.01)$. There was no significant correlation between Identity Confusion and the two earlier phases of Individual Sexual Identity. Thus, for women Hypothesis 3a was partially supported in that identity confusion correlated in the negative direction with the two later phases of Individual Sexual Identity (i.e., Deepening/Commitment and Internalization/Synthesis).

For men, there was a negative and significant correlation between Identity Confusion and the two later phases of Individual Sexual Identity. The correlation between the Individual Deepening/Commitment subscale and Identity Confusion was $.44(p<.01)$ and the correlation between the Individual Internalization/Synthesis subscale and Identity Confusion was $-.51(p<.01)$. Regarding the two earlier phase of Individual Sexual Identity, there was a significant correlation between Identity Confusion and Individual Exploration $(r=-.37, p<.05)$, however this correlation was in the negative direction. There was no significant correlation between the Individual Awareness subscale and Identity Confusion. Thus, for men Hypothesis $3 \mathrm{~b}$ was partially supported in that identity confusion correlated in the negative direction with the two later phases of Individual Sexual Identity (i.e., Deepening/Commitment and Internalization/Synthesis).

No predictions were made about the relationship between Identity Confusion and the Group Membership Identity subscales; however for both women and men there was a positive and significant correlation between Identity Confusion and Group Awareness (women; $r=.42, p<.05$, men; $r=.48, p<.01$ ). Further, there was negative and significant correlation between Identity Confusion and Group Internalization/Synthesis 
for both women and men (women; $r=-.36, p<.05$, men; $r=-.60, p<.01$ ). These results provide additional support for the theoretical relationship between sexual minority identity formation and identity related experiences unique to sexual minority individuals such as identity confusion.

Hypothesis 3b: Internalized Homonegativity.

1. Internalized Homonegativity will be positively and significantly correlated with the two earlier phases of Individual Sexual and Group Membership Identity (Awareness and Exploration).

2. Internalized Homonegativity will be negatively and significantly correlated with the two later phases of Individual Sexual and Group Membership Identity (Deepening/Commitment and Internalization/Synthesis).

For women, there was a positive and significant correlation between Internalized Homonegativity and Group Awareness. The correlation between the Group Awareness subscale and Internalized Homonegativity was .52 $(p<.01)$. Moreover, there was a negative and significant correlation between Internalized Homonegativity and Individual Internalization/Synthesis as well as Group Internalization/Synthesis. The correlation between the Individual Internalization/Synthesis subscale and Internalized Homonegativity was -.50 $(p<.01)$ and the correlation between the Group Internalization/Synthesis subscale and Internalized Homonegativity was -.60 $(p<.01)$. There was no significant correlation between Internalized Homonegativity and Individual Awareness, Individual Exploration, Individual Deepening/Commitment as well as Group Exploration and Group Deepening/Commitment. Therefore, Hypothesis 3b was partially 
supported for women in that Internalized Homonegativity correlated positively with Group Awareness and negatively with Individual and Group Internalization/Synthesis. For men, there was a positive and significant correlation between Internalized Homonegativity and Individual Awareness as well as the two earlier phases of Group Membership Identity. The correlation between the Individual Awareness subscale and Internalized Homonegativity was $.55(p<.01)$. The correlation between the Group Awareness and Group Exploration subscales were $.69(p<.01)$ and $.37(p<.05)$ respectively. Moreover, there was a negative and significant correlation between Internalized Homonegativity and the two later phases of Individual Sexual Identity. The correlation between the Individual Deepening/Commitment subscale and Internalized Homonegativity was $-.52(p<.01)$ and the correlation between the Individual Internalization/Synthesis subscale and Internalized Homonegativity was -.64 $(p<.01)$. There was no significant correlation between Internalized Homonegativity and the Individual Exploration and Group Deepening/Commitment subscales. Therefore, Hypothesis $3 \mathrm{~b}$ was partially supported for men in that there was a positive correlation between Internalized Homonegativity and Individual Awareness as well as Group Awareness and Group Exploration. Further, there was a negative correlation between Internalized Homonegativity and Individual Deepening/Commitment and Internalization/Synthesis.

Hypothesis 3c: Same Group Orientation.

1. Same Group Orientation will be negatively and significantly correlated with the two earlier phases of Group Membership Identity (Awareness and Exploration). 
2. Same Group Orientation will be positively and significantly correlated with the two later phases of Group Membership Identity (Deepening/Commitment and Internalization/Synthesis).

For women, there was a negative and significant correlation between Same Group Orientation and the Group Awareness subscale. The correlation between the Group Awareness subscale and Same Group Orientation was -.40 $(p<.05)$. Moreover, there was a positive and significant correlation between Same Group Orientation and the two later phases of Group Membership Identity. The correlation between the Group Deepening/Commitment subscale and Same Group Orientation was $.41(p<.05)$ and the correlation between the Group Internalization/Synthesis subscale and Same Group Orientation was .68 $(p<.01)$. There was no significant correlation between Same Group Orientation and the Group Exploration subscale. Thus, Hypothesis 3c was partially supported for women in that there was a negative correlation between Same Group Orientation and Group Awareness and a positive correlation between Same Group Orientation and Group Deepening/Commitment and Internalization/Synthesis

For men, there was a negative and significant correlation between Same Group Orientation and the Group Awareness Subscale. The correlation between the Group Awareness subscale and Same Group Orientation was -.45 $(p<.05)$. Moreover, there was a positive and significant correlation between Same Group Orientation and the Group Internalization Synthesis subscale. The correlation between the Group Internalization/Synthesis subscale and Same Group Orientation was $.55(p<.01)$. There was no significant correlation between Same Group Orientation and the Group Exploration and Group Deepening/Commitment subscales. Thus, Hypothesis 3c was 
partially supported for men in that, for Same Group Orientation, there was a negative relationship with Group Awareness and positive relationship with Group Internalization/Synthesis.

\section{Hypothesis 3d: Outness}

1. Outness will be negatively and significantly correlated with the two earlier phases of Group Membership Identity (Awareness and Exploration).

2. Outness will be positively and significantly correlated with the two later phases of Group Membership Identity (Deepening/Commitment and Internalization/Synthesis).

For women, there was a negative and significant correlation between Outness the Group Awareness subscale. The correlation between the Group Awareness subscale and Outness was $-.56(p<.01)$. Moreover, there was a positive and significant correlation between Outness and the Group Internalization/Synthesis subscale. The correlation between the Group Internalization/Synthesis subscale and Outness was .42 $(p<.05)$. There was no significant correlation between Outness and the Group Exploration and Group Deepening/Commitment subscales. Thus, Hypothesis 3d was partially supported for women in that, for Outness, there was a negative relationship with Group Awareness and a positive relationship with Group Internalization/Synthesis.

For men, there was no significant correlation between Outness and any of the four phases Group Membership Identity, therefore Hypothesis 3d was not supported for men.

No predictions were made about the relationship between Outness and the Individual Sexual Identity subscales, however for women there was a negative and significant correlation between Outness and the Individual Awareness subscale $(r=-.37$, $p<.01)$ Further, there was a positive and significant correlation between Outness and 
Individual Internalization/Synthesis for women $(r=.42, p<.05)$. These results are intriguing given that for men, Outness did not correlate significantly with any of the four phases of both Individual Sexual and Group Membership Identity.

Hypothesis 4: Discriminant validity: The eight subscales will not correlate significantly with dogmatism. As shown in Tables 4 and 5, there was no significant correlation between the Dogmatism scale and the following subscales of the SSOIQ for both samples of women and men; Individual Exploration, Individual Deepening/Commitment, Individual Internalization/Synthesis, Group Awareness, Group Exploration, and Group Internalization Synthesis. For both women and men, there was a positive and significant correlation between Individual Awareness and Dogmatism (women; $r=.46, p<.05$, men; $r=.52, p<.01$ ). Further, for men there was a positive and significant correlation between Group Deepening/Commitment and Dogmatism $(r=.53$, $p<.01)$. Thus, hypothesis 4 was partially supported. The results for women suggest a distinction between one's movement in an identity formation process and closed cognitive structure with the exception of early awareness. For men, the results suggest that the extent to which one is rigid in his belief system is somewhat related to his ability to move through a fluid identity process. 


\section{Chapter 5: Discussion}

In the current work, the reliability and validity of a measure of sexual minority identity formation (the Same-Sex Orientation Identity Questionnaire; SSOIQ) was assessed with a racially/ethnically diverse sample. The SSOIQ was developed to measure one's location in a sexual minority identity formation process. The measure was derived from the Fassinger and colleagues (Fassinger, 2001a,b; Fassinger \& Miller, 1996; McCarn \& Fassinger, 1996) dual-trajectory model that hypothesizes two separate but reciprocal processes of individual sexual identity development and group membership identity development in a four-phase developmental sequence. First, the items in two existing versions of the measure (a women and men's version) were revised to better capture current understandings of the experiences of sexual minority people of color. Next, a small pilot study was conducted employing a card-sort methodology with a sample of 10 diverse sexual minority people of color. Results from the pilot study indicated support for the model and suggested that the measure was appropriate for the implementation of the web-based validation study. Estimates of internal consistency reliability were assessed through Cronbach's alpha. A preliminary evaluation of the theoretical model underlying the measure was conducted by examining the interrelationships of the conceptually distinct phases of the model. Finally, convergent validity was partially established through relationships of the two versions of the measure to measures of identity confusion, internalized homonegativity, same group orientation, and outness. Discriminant validity was partially established using a measure of dogmatism.

Summary of Findings and Comparison to the Existing Literature 
In the current study, it was hypothesized that the eight subscales of the SSOIQ for both the women and men's version would demonstrate adequate levels of internal consistency reliability with the current sample. This hypothesis was partially supported in that only two of the eight subscales (i.e., Individual Exploration and Individual Internalization/Synthesis) produced an adequate estimate of internal consistency reliability for the sample of women and one of the eight subscales (i.e., Individual Internalization/Synthesis) produced an adequate level of internal consistency reliability for the sample of men. The reliability estimates ranged from .14 to .77 for women and .26 to .71 for men. The ranges obtained in the current study are somewhat consistent with what has been found in previous studies. Porter (1998) reported Cronbach's Alpha coefficients for each of the eight subscales with a sample of 55 lesbian women. The alpha levels reported in Porter's study ranged from .57 to .79 in the individual identity subscales and .53 to .73 in the group identity subscales. Further, with a sample of 192 undergraduate lesbian women, Tomlinson and Fassinger (2003) reported alphas for the individual subscales ranging from .76 to .88 and the group subscales ranging from .61 to .72. One possible reason for the weak level of internal consistency reliability obtained in the current study concerns the small sample sizes $($ women $=32$, men $=37)$. Coefficient alpha is greatly influenced by the number of items in a given scale as well as the size of the sample (Pett et al., 2003). To test if increasing the number of items would affect the alpha level, the alpha for the full scale individual and group branches (20 items each) were determined. The Individual full scale alpha coefficient for women demonstrated an adequate level of reliability (alpha $=.77$ ). For men, the Individual and Group full scale alphas approached and adequate level of reliability (.68 and .65 respectively). Thus, given 
the alpha levels reported in previous research and the limitations of Cronbach's alpha, it is possible that increasing the sample size substantially will produce adequate levels of internal consistency reliability. Alternatively, researchers conducting further psychometric work with this measure might consider the relevance of assessing the internal consistency of the current subscales. The items within each of the eight subscales were designed to capture cognitive, emotional, and behavioral experiences within a particular phase od development. However, these experiences may not necessarily cooccur, thus examining the internal consistency of these events is possibly unwarranted.

From the preliminary evaluation of the measure based on the intercorrelations between the subscales, partial support was obtained for the theoretical model. That is, a negative and significant correlation between the Awareness and Internalization/Synthesis subscales in the group branch was found for men. Also for men, a positive and significant correlation between three of the four parallel phases (i.e., Awareness, Exploration, and Internalization/Synthesis) was obtained. For the women's measure, however, a positive and significant correlation was found only between the parallel Awareness and Internalization/Synthesis phases. The discrepant findings between the women and men's version of the measure possibly implicate gender differences in the process of sexual minority identity development. For men, it seems that the phases are interrelated in the expected directions. For women, however, most of the phases were not interrelated at a statistically significant level. Alternatively, it is also possible that the small sample of women $(\mathrm{N}=32)$ prevented the discovery of a significant relationship between the subscales. The sample size required to provide sufficient power to detect a true effect was 33. Further, two of the correlations approached significance based off a p value that fell 
very close to the .05 cut off. Although no conclusions can be made about these nonsignificant findings, it is imperative to retest this hypothesis with a larger sample of women. In a sample of 192 undergraduate lesbian women, Tomlinson and Fassinger (2003) conducted an assessment of the Pearson coefficients and confirmed that each identity phase exhibits stronger correlations with the phase closest to it, and the magnitude of the correlations decrease moving outward. The first and final phases (i.e., Awareness and Internalization/Synthesis) within both subscales were observed to be negatively correlated.

Cohen (1994) emphasized the importance of extending research beyond the significant-non-significant dichotomy. It was suggested by Cohen that strict adherence to a $\mathrm{p}$ value of .05 restricts knowledge in that it categorizes potentially important findings as non-significant. For this reason, Cohen argued that researchers should give weight to the size of an effect or result. Thus, interpreting the non-significant correlations with regards to effect sizes (i.e., a measure of the magnitude of the effect or the degree to which the effect exists) provides additional support for the model. In determining the size of an effect, the following guidelines were used as suggested by Cohen: A correlation of .15 was interpreted as a small effect, .3 a medium effect and .5 a large effect. For the women's version of the measure, the correlation between the Individual and Group Exploration phases was .21. Also, the correlation between the Individual and Group Deepening/Commitment phases was .29. While both these correlations did not reach significance at the .05 level, they do represent a medium effect. Similarly, in the women's version of the measure, the correlations between Individual Awareness and Internalization as well as Group Awareness and Internalization were -.25 and -.28 
respectively. Again, while these correlations did not reach significance they demonstrate a medium effect. For the men's version of the measure, a small effect between the Individual and Group Deepening phases was determined with a correlation of .16. Further, a medium effect between the Individual Awareness and Internalization phases was determined with a correlation of -.25 .

Overall, results from the current study suggest that the subscales within the individual and group branches were measuring overlapping, and progressively changing constructs. Also, the parallel phases for both versions of the measure were not too highly correlated demonstrating the expected amount of convergence. This suggests that the phases within the Individual and Group trajectories capture two distinct processes of sexual minority identity development as postulated by Fassinger and colleagues. Further, the findings for both women and men provide stronger support for the beginning and ending phases. These results are consistent with the larger literature on social group identity development that suggests that development is more clearly demarcated at the polar ends with less clarity during transition or middle phases.

The current study hypothesized that SSOIQ would demonstrate convergent validity by correlating in expected directions with the following constructs; identity confusion, internalized homonegativity, same group orientation, and outness. Identity confusion correlated in the negative direction with the two later phases of individual sexual identity (i.e., Deepening/Commitment and Internalization/Synthesis) for both women and men. The relationship between identity confusion and the two later phases of individual sexual identity indicates that the commitment and self-acceptance of one's same-sex orientation converges with the questioning and feelings of bewilderment 
implied in identity confusion. The lack of convergence as expected between identity confusion and the two earlier phases of individual sexual identity suggests that this process (i.e., identity confusion) is not exactly parallel to individual awareness and exploration or, alternatively, the items in the measure did not adequately capture the experiences related to the construct of identity confusion.

The relationship between internalized homonegativity and the various phases of the model were different for women and men. For women, internalized homonegativity correlated positively with Group Awareness and negatively with Individual and Group Internalization/Synthesis. For men, there was a positive correlation between internalized homonegativity and Individual Awareness as well as Group Awareness and Group Exploration. Further, there was a negative correlation between internalized homonegativity and Individual Deepening/Commitment and Internalization/Synthesis. Thus, for men it seems that internalized homonegativity as a construct provided more solid evidence of convergent validity. This finding is intriguing in light of research pointing to gender differences in socialization experiences pertaining to same-sex sexuality. Men, particularly in African American and Latino cultures, are socialized to adhere to strict standards of masculinity that accentuate heterosexuality (Zea et al., 2003). These social norms denigrate and scorn sexual intimacy between men. By comparison, women are allowed more fluidity in their sexuality and the social sanctions against samesex intimacy between women are less severe (Chivers et al., 2004; Parks et al., 2004). Given this, perhaps internalized homonegativity is a construct germane to the process of sexual minority identity development for men, yet less so for women. 
In regards to same group orientation, for women, there was a negative correlation between same group orientation and Group Awareness and a positive correlation between Group Deepening/Commitment and Internalization/Synthesis. For men, there was a negative correlation with Group Awareness and positive correlation with Group Internalization/Synthesis. Thus, support for the validity of the group membership phases was found for the Awareness and Internalization/Synthesis phases in both the women and men's version of the measure while less support was found for the middle phases. This finding is consistent with recent work by Phinney \& Ong (2007) suggesting that the process of developing a same group orientation to racial/ethnic identity groups contains only a two factor structure; these factors were interpreted and labeled as Exploration and Commitment.

Convergent validity between the SSOIQ and outness was partially supported for women in that there was a negative relationship with Group Awareness and a positive relationship with Group Internalization/Synthesis. For men, however, there was no significant correlation between outness and any of the four phases of group membership identity. The gender differences in the relationship between outness and the SSOIQ raise questions about the differential experiences in the process of coming out between sexual minority women and men and the implications of these gendered experiences for identity formation. Further, these findings are consistent with previous research noting the racial/ethnic differences in disclosure of sexual orientation between minority and majority individuals (Rosario et al., 2004). Rosario et al. suggest that the process of identity integration is somewhat delayed and not directly linked to disclosure for Black and Latino youths when compared to White youths. 
In addition to convergent validity, a measure of dogmatism was used to establish discriminant validity. For women, the results suggest a distinction between one's movement in an identity formation process and dogmatic belief system, with the exception of early awareness. Thus, perhaps for women, having a closed cognitive system is solely related to the onset or burgeoning of a minority sexuality. For men, the results suggest that the extent to which one is rigid in his belief system is somewhat related to his ability to move through a fluid identity process. Support for this was found both at the early awareness phase and the deepening/commitment phase. Thus, perhaps dogmatism represents a discriminant construct for women, yet not for men. Strengths and Limitations of the Study

A primary strength of the current work is that the measure undergoing evaluation has been strongly rooted in theory. The items pertaining to the eight distinct subscales of the measure were developed and refined based on what scholars in the identity literature have noted as a sophisticated developmental theory of sexual minority identity formation (Worthington, Navarro, Bielstein-Savoy, \& Hampton, 2008). Although numerous theoretical models of sexual minority identity development exist, there are few that have undergone a formal validation process. A primary reason for this lack of empirical scrutiny is the absence of measures that correspond to a particular theory. Provided the current findings hold through further data collection, the preliminary evaluation of the SSOIQ lays a solid foundation for future research testing the structural validity of the model. In relation, the detailed work that went into revising and piloting the items is another merit of the study. 
A second strength of the current work is the development of two different versions of the measure, one for women and a separate for men. Discrepant findings were obtained with regard to the convergent and discriminant constructs. These findings are interpretable within the larger literature highlighting gender differences in sexuality and might have gone unexamined had the measures, and subsequent analyses, not been separated.

In addition, a strength unique to the measure developed in the current work is its availability for use with persons of various minority sexual orientations. This inclusiveness allows researchers to use the measure without requiring participants to specify a sexual identity label. Researchers can then study broader questions about samesex sexuality without the constraints inherent in temporally bound sexual identity labels (Bieschke et al., in press; Worthington et al., 2007).

Several limitations of the current study should be noted. First, it is possible that the current results have been influenced by sampling bias. As is common of the work in this area, the sample in the current work consisted of conveniently available volunteer respondents rather than a randomly selected representative sample extracted from a clearly-defined population. This sampling approach is more likely to solicit the participation of individuals who are intrinsically interested in research on the topic of sexuality. However, given that the current study explored a relatively new area of inquiry with an exceedingly difficult to reach population, sampling bias issues were somewhat unavoidable. Also, because there have been so few empirical studies published on sexual minority persons of color, there is a dearth of information from which to make any claims about the population. To address this limitation, future research calls for the usage of 
large, randomized samples as opposed to convenience samples. A large randomized sample would provide more variability in sexual orientation.

Another sampling concern is that the response rate is unknown. Due to the online nature of the study and the snowball sampling technique employed, the actual number of eligible participants who chose not to participate in the study was indeterminable. This limitation is consistent with the larger body of research on sexual minority persons of color. This also points to a drawback of online survey research. However, because this study attempted to explore a sensitive topic on an underserved population, using an online data collection process the both protected participants' anonymity and extended beyond racially and ethnically homogenous sexual minority communities was critical. Furthermore, even with the conveniences inherit in online data collection, this population proved incredibly difficult to reach.

A second limitation of the current study and consistent with the literature on sexual minority persons is that identity representations in the later phases were somewhat overrepresented in the sample. Sexual minority persons who are more integrated in their identity and have disclosed their identity to others despite possible stigma are presumably more likely to respond to recruitment material. Hence, snowball sampling and strategically recruiting from venues, organizations, social groups and the like that do not specifically target sexual minority persons represented an attempt to reach those in early identity phases, as well as those who have not self-disclosed. This limitation calls to mind a more philosophical question of whether an identity that has not yet been formed or crystallized can be studied empirically. 
The current study intended to be inclusive of culturally diverse experiences in the sexual minority identity formation process; however the instruments were only available in English. Given this limitation, the experiences of non English speaking sexual minority persons were not considered in the current work. Translating the instruments and obtaining evidence of reliability and validity is thus a necessary area for future research. Such research would contribute greatly to the literature by bringing the experiences of a truly understudied population to the forefront.

The current study intended to evaluate an inclusive model of sexual minority identity formation with a sample of racial/ethnic minority persons. Thus, the sample in the current study was not representative of the general population of sexual minority persons. Evaluating a theoretical model with a sample that is not representative of the population of interest has implications for the generalizability of the model. The model under evaluation is thought to be applicable to the experiences of all individuals who represent a sexual minority identity. However, the focus of the current work was on racial/ethnic minority persons because their experiences are relatively understudied in the literature. A second study would then be appropriate to provide validity evidence for a more representative sample and further contribute to the literature. A fundamental aspect of the model is that it be inclusive of the experiences of people of color, thus it seems appropriate to test for cultural inclusiveness prior to testing the model on individuals from the majority racial/ethnic culture. Although there are methodological limitations that come from placing all people of color into one group, this approach is justified on the grounds that racial/ethnic minority individuals in the United States encounter similar (although not identical) experiences of oppression (Helms, 1992). 
A final key consideration of the current study is that the model, as well as the instrument under evaluation, are temporally limited. The theoretical model that provided the underpinnings of the instrument was developed over a decade ago. Further, participants in the preliminary validation samples represented identity development processes that, at that time, had occurred two or more decades previously. In light of current thinking regarding sexual minority identity enactment (Fassinger \& Arseneau, 2007), temporal context is a critical aspect of one's developmental process. One attempt made to address the relevance of the instrument to the present was revising the items based on current thinking and then submitting the items to a pilot study with individuals from a relatively young cohort.

\section{Implications for Research}

Findings from the current study provide several implications for research. One direction for future research is to conduct a confirmatory factor analysis (CFA) on the SSOIQ. CFA is a measurement tool used to confirm that a hypothesized factor structure, specified a priori, provides a good fit to a given data set (Kahn, 2006). In CFA, hypotheses are derived from a theory postulating the relationships among constructs and observed variables. The interrelationships among the eight subscales or phases in the model provide preliminary evidence for the theoretical framework on which the measure was developed. Thus, further research designed to formally test the structural links from latent factors to measured variables (i.e., items) is needed. The fundamental research question underlying this investigation is the extent to which the theory of sexual minority identity formation is plausible with a sample of people of color. Questions of this kind are more directly addressed utilizing CFA procedures. 
Another area for future research is to directly test the theoretical model presented in the current work against competing models that are equally plausible. Building off the work of Fassinger and colleagues (Fassinger \& Miller, 1996; McCarn \& Fassinger, 1996), Worthington et al. (2008) have recently developed a multidimensional measure of sexual identity exploration and commitment. In their model, the authors present four distinct factors assessing commitment, exploration, sexual orientation identity uncertainty, and synthesis/integration. This multidimensional model is nested within the model evaluated in the current work. However, a key difference between this model and the model evaluated in the current study is that the Worthington et al. model is applicable to heterosexual as well as sexual minority persons. The model presented by Fassinger and colleagues, on the other hand, considers the process of developing a stigmatized individual and social group identity as distinct from developing a majority identity. In the current model, the context of oppression and stigmatization is considered central to the process of sexual identity development for sexual minority individuals. Direct comparisons between competing hypothetical models are needed; that is, a model formulated on the universality of sexual identity development versus one that focuses solely on the shared experiences of those developing a marginalized identity. Research of this nature would not only advance the literature on sexual minority identity formation but the much broader literatures on sexuality and social group identity development.

Finally, provided subsequent work is conducted to more firmly establish the reliability and validity of the SSOIQ with larger samples, this measure could be used in cross-sectional research comparing the process of sexual identity formation for 
individuals who adopt different identity labels (e.g., is this process the same for bisexual and lesbian individuals?).

\section{Implications for Practice}

Reynolds and Hanjorgiris (2000) have noted that sexual minority individuals seek therapy in greater numbers than their heterosexual counterparts. Given the overrepresentation of sexual minority persons in the population of those who seek mental health services, sexual minority identity formation frameworks have direct implications for practice. Having an appropriate knowledge of identity development issues that pertain to sexual minority individuals over the course of the life span is a critical aspect of providing affirmative and ethical services. Moreover, the way one comes to label her- or himself is considered to be a relatively important aspect of self in contemporary America. Developmental models provide a language for practitioners to use when describing or empathizing with the shared experiences of sexual minority clients (Fassinger, 2000). Further, with developmental frameworks in mind, counselors are better equipped to foresee or predict experiences related to developing and/or enacting a minority identity (McCarn \& Fassinger, 1996). Research that delineates this process provides counselors with a framework from which to normalize the identity related experiences of sexual minority individuals.

In addition, the preliminary evaluation of the model conducted in the current work suggests that the development of an internal sense of self operates on a different trajectory then the development of a group identity. This information challenges counselors to think critically about encouraging their sexual minority clients to explore and affiliate with sexual minority communities. This sort of encouragement might hastily 
rush clients along a complex developmental process. In facilitating the identity formation of sexual minority clients, counselors must be prepared to assist clients in identity management, serve as a source of support, confront internalized homonegativity/homophobia, and appreciate the difficulties involved in the process of self-disclosure (Reynolds \& Hanjorgiris, 2000).

To conclude, results from the current study point to the complexities of the identity related experiences shared by sexual minority individuals. Pursuing research and clinical work from an inclusive and comprehensive framework will better prepare researchers and practitioners to conduct research and provide services that truly resonate with the lived experiences of individuals who represent the diverse sexual minority community (Bieschke et al., in press). 
APPENDIX A: ITEM REVISION WORKSHEET

\begin{tabular}{|c|c|c|c|c|c|}
\hline Phase & $\begin{array}{c}\text { Item } \\
\#\end{array}$ & Code(s) & $\begin{array}{c}\text { Keep, } \\
\text { Eliminate, } \\
\text { Revise }\end{array}$ & Comment & Revision \\
\hline $\begin{array}{l}\text { Individual: } \\
\text { Awareness }\end{array}$ & 1 & & & & \\
\hline & 2 & & & & \\
\hline & 3 & & & & \\
\hline & 4 & & & & \\
\hline & 5 & & & & \\
\hline $\begin{array}{l}\text { Individual: } \\
\text { Exploration }\end{array}$ & 1 & & & & \\
\hline & 2 & & & & \\
\hline & 3 & & & & \\
\hline & 4 & & & & \\
\hline & 5 & & & & \\
\hline $\begin{array}{l}\text { Individual: } \\
\text { Deepening/ } \\
\text { Commitment }\end{array}$ & 1 & & & & \\
\hline & 2 & & & & \\
\hline & 3 & & & & \\
\hline
\end{tabular}




\begin{tabular}{|c|c|c|c|c|c|}
\hline & 4 & & & & \\
\hline & 5 & & & & \\
\hline Phase & $\begin{array}{c}\text { Item } \\
\#\end{array}$ & Code(s) & $\begin{array}{c}\text { Keep, } \\
\text { Eliminate, } \\
\text { Revise }\end{array}$ & Comment & Revision \\
\hline $\begin{array}{l}\text { Individual: } \\
\text { Internalization/ } \\
\text { Synthesis }\end{array}$ & 1 & & & & \\
\hline & 2 & & & & \\
\hline & 3 & & & & \\
\hline & 4 & & & & \\
\hline & 5 & & & & \\
\hline $\begin{array}{l}\text { Group: } \\
\text { Awareness }\end{array}$ & 1 & & & & \\
\hline & 2 & & & & \\
\hline & 3 & & & & \\
\hline & 4 & & & & \\
\hline & 5 & & & & \\
\hline $\begin{array}{l}\text { Group: } \\
\text { Exploration }\end{array}$ & 1 & & & & \\
\hline
\end{tabular}




\begin{tabular}{|l|c|c|c|l|l|}
\hline & 2 & & & & \\
\hline & 3 & & & & \\
\hline & 4 & & & & \\
\hline Phase & Item & Code(s) & $\begin{array}{c}\text { Keep, } \\
\text { Eliminate, } \\
\text { Revise }\end{array}$ & Comment & \\
\hline $\begin{array}{l}\text { Group: } \\
\text { Deepening/ } \\
\text { Commitment }\end{array}$ & 1 & & & & \\
\hline & 2 & & & & \\
\hline & 3 & & & & \\
\hline & & & & & \\
\hline Synthesis & 1 & & & & \\
\hline & 5 & & & & \\
\hline & & & & & \\
\hline & & & & \\
\hline
\end{tabular}




\begin{tabular}{|l|l|l|l|l|l|}
\hline & 5 & & & & \\
\hline
\end{tabular}

\section{Codes:}

$\mathrm{C}=$ Cognition: Thoughts pertaining to perception, introspection, belief, or volition $\mathrm{E}=$ Emotion: Affective reaction or experience

$\mathrm{B}=$ Behavior: Action(s)

$\mathrm{K}=$ Knowledge: Information

$\mathrm{R}=$ Relational: Interpersonal interaction

$\mathrm{CC}=$ Cultural Context: Circumstances or conditions surrounding an experience pertaining to culture

General Themes to Consider:

- Labeling/naming/language

- Sexual behavior vs. identity

- Conflation of gender and sexual orientation

- Temporal influence

Themes Unique to Racial/Ethnic Minorities:

- Conflicting allegiances

- First identity, race/ethnicity or sexual orientation? "invisible middle"

- Religion as a place of connectedness and home

- Family and community acceptance

- Dating and sex (who do I date?)

- Role models and support networks

- Similarities and differences between racism and heterosexism

- Silence around sexual orientation as racial oppression takes precedence

- Friction with white LGB persons; owning "whiteness" before "gayness"

- Culture specific notions of gender

- Invisibility of sexuality, particularly for women in patriarchal cultures

- Invisibility of communities and identities organized around sexual orientation 


\section{APPENDIX B: CARD SORT INSTRUCTIONS}

\section{Card Sort Procedure and Instructions}

Introduction: Begin by thanking participants for their willingness to volunteer their time and share their experience. My advisor and I are interested in how people come to form various aspects of their identity. For this study in particular, we are interested in the identity process related to sexuality for people of color. We have a theoretical framework for the process of sexual identity development and we want to see if our model fits for people of color. Today I will be asking you to complete two card sort activities. We will then close with a discussion about what the card sort process was like for you. Please make notes of your thoughts as you go through the procedures and we can discuss them when we are finished with both activities.

Sorting Procedure A: Participants will sort items into eight piles prior to receiving description of identity formation model.

Introduction: We will now begin with the first procedure. You have been given a set of statements intended to reflect aspects of the process of developing an identity as a "sexual minority;" that is, someone who is attracted to or oriented to people of one's same gender either part of the time or all of the time, or someone who might consider this possibility in his or her life.

Task (1) I would like you to begin by taking your 40 cards and separating them into two groupings of 20 cards each. Please group the cards in a way that makes sense to you based on content. Please select on pile as Group A and the other pile as Group B, write the card numbers for each pile on your handout under step one.

Task (2) Next, sort each of the two large groups (A and B) into four piles with five cards in each pile. Please group the statements in a way that makes sense to you based on content and write a label, brief explanation, and the card numbers for each pile on your handout under step two.

Task (3) Now, please place each of the four piles within each of the two larger groups in sequential order. Please write the labels you provided in step two in the order you have determined. Also provide an explanation of your sequencing on your handout. You do not need to place the five items in each of the eight piles in any particular order.

Task (4) Finally, please take a few minutes to jot down any thoughts or reactions you have about this sorting task or your groupings under step four.

Sorting Procedure B: Participants will sort items into groupings and piles as they receive a description of the model.

Introduction: Thank you for completing the first card sort activity. Now I will ask you to try and erase your groupings and thoughts from your mind as we begin the second card 
sort activity. In the first sorting procedure we were interested in what groupings you came up with on your own. Now in the second card sort activity I will provide more structure based on our model as you sort the cards. You have been given the same set of statements as in the first sorting exercise. Again, these statements are intended to reflect aspects of the process of sexual minority identity formation.

Task (1) Please begin by sorting all of the statements into two separate equal size groups. One group will be labeled "Individual Sexual Identity" and the other group will be labeled "Group Membership Identity" (have participants write headings on their handout under step one). Please group the statements in a way that makes sense to you based on a match between the content of the statement and the description provided for each of the two groupings.

Individual Sexual Identity: Statements under this heading represent the internal process of understanding and coming to terms with one's own sexual preferences in terms of thoughts, feelings, and behaviors.

Group Membership Identity: This process is thought to be parallel to the process of individual sexual identity formation (note that it is not necessarily simultaneous to this process). Statements in this pile should reflect the experience of being a person with those sexual preferences in this society at this time. That is, thoughts, feelings, and behaviors about a reference group that represents "sexual minorities."

Write the card numbers that correspond with each of your groupings on your handout under step one.

Task (2) Next, take the Individual Sexual Identity Pile and set the other aside. Please sort these cards into four piles of five cards each that seem to go together in a way that captures the flow of developing a sexual identity that is a sequencing of feelings, thoughts, and behaviors in forming this identity overtime. Please use your own knowledge and experience to guide your thinking.

Task (3) Please provide the card numbers, a label, and short descriptor of each pile that you've created on your handout under step two. Also, please indicate which pile best represents where you think you are in your own process of development at this point in time by circling that pile label.

Task (4) Now, please take the Group Membership Identity Pile and sort the cards in four piles of five cards each that seem to go together in a way that captures the flow of developing an awareness of and connection to a reference group regarding sexual identity.

Task (5) Please provide the card numbers, a label, and short descriptor of each pile that you've created on your handout under step two. Also, please indicate which pile best represents where you think you are in your own process of development at this point in time by circling that pile label. 
Task (6) Finally, please take a few minutes to jot down any thoughts or reactions you have about this sorting task or your groupings under step three.

\section{Discussion}

Introduction: Thank participants for completing card sort activity. Before we begin, I would like ask for your permission to tape record our discussion. The tape will remain in my possession and will only be listened to by myself and my advisor. I am now going to ask you a sequence of questions that mirrors the tasks we did. We will begin with procedure $\mathrm{A}$ and then move on to procedure $\mathrm{B}$, please feel free to use your handout as a reference.

Discussion Questions:

1.) General reactions to the first card sort process.

What was difficult?

What was easy?

Was anything confusing?

How satisfied were you with your piles?

What did the first sorting process capture?

2.) Reactions to the second card sort process.

Did the additional structuring help or hinder the task for you?

What was it like to sort into Individual and Group piles?

What was it like to sort each of the large piles into four sequential piles?

For you, what did the second sorting process capture?

3.) Reactions to choosing two piles (one for Individual and one for Group) that represent your own development?

4.) Feedback on item content.

\section{Closing}

(1) Ask participants to complete demographic questionnaire.

(2) Invite participants to contact the researchers if they have further questions or concerns. 


\section{APPENDIX C: CARD SORT HANDOUT}

Participant Code (last 4 digits of social):

Procedure A

Step 1

\section{Group A}

List Card \#'s

\section{Group B}

List Card \#'s

Step 2

\begin{tabular}{|l|l|}
\hline Group A & Group B \\
\hline Pile 1_Label: & Pile 1_Label: \\
& \\
List Card \#'s: & List Card \#'s: \\
\hline Pile 2_Label: & Pile 2_Label: \\
& \\
& \\
List Card \#'s: & \\
\hline Pile 3_Label: & List Card \#'s: \\
& Pile 3_Label: \\
& \\
\hline List Card \#'s: & \\
\hline
\end{tabular}




\begin{tabular}{|l|l|}
\hline Pile 4_Label: & Pile 4_Label: \\
& \\
& \\
List Card \#'s: & List Card \#'s: \\
\hline
\end{tabular}

\section{Step 3}

\begin{tabular}{|c|c|}
\hline Group A & Group B \\
\hline Label \#1: & Label \#1: \\
\hline List Card \#'s: & List Card \#'s: \\
\hline Label \#2: & Label \#2: \\
\hline List Card \#'s: & List Card \#'s: \\
\hline Label \#3: & Label \#3: \\
\hline List Card \#'s: & List Card \#'s: \\
\hline
\end{tabular}




\begin{tabular}{|l|l|}
\hline Label \#4: & Label \#4: \\
& \\
List Card \#'s: & List Card \#'s: \\
\hline
\end{tabular}

\section{Step 4}

Comments, thoughts, reactions: 
Participant Code:

Procedure B

Step 1

Group A

List Card \#'s

Group B

List Card \#'s

\section{Step 2}

\begin{tabular}{|l|l|}
\hline Group A & Group B \\
\hline Label \#1: & Label \#1: \\
& \\
& \\
List Card \#'s: & List Card \#'s: \\
\hline Label \#2: & Label \#2: \\
& \\
\hline List Card \#'s: & \\
\hline Label \#3: & \\
& \\
\hline & List Card \#'s: \\
\hline & \\
\hline & \\
\hline
\end{tabular}




\begin{tabular}{|l|l|}
\hline List Card \#'s: & List Card \#'s: \\
& \\
\hline Label \#4: & Label \#4: \\
& \\
List Card \#'s: & \\
\hline
\end{tabular}

\section{Step 3}

Comments, thoughts, reactions: 


\section{APPENDIX D: RECRUITMENT ADVERSTISEMENT \\ ONLINE SURVEY_ENTER TO WIN \$50 GIFT CARD}

We are a diverse team of researchers at the University of Maryland, College Park conducting a study on the life experiences of People of Color with Same-Sex Attractions. If you identify as a person of color or racial/ethnic minority and as any of the following; lesbian, gay, bisexual, queer, questioning, bi-curious, same-gender loving, exploring same-sex sexuality, or have some same-sex attraction or orientation then we are interested in your experience! Participation involves the completion of several questionnaires and will take approximately 20-30 minutes. You must be at least 18 years of age to participate.

After completing the survey, you may enter a drawing for one of two \$50 Target gift cards. Your participation in this study is entirely voluntary and you can decide to stop at any time. Your responses will remain confidential and no identifying information will be requested. You will only be asked for your email address for the purposes of entering the drawing. Entry into the drawing is entirely optional.

If you are interested in participating in this study please visit the website listed below:

https://www.psychdata.com/s.asp?SID=122739

Or go to www.psychdata.com and enter the number 122739 where it reads "Go to survey \#"

Your participation will be greatly appreciated! If you choose not to participate, please consider passing this information on to others who may be interested. Thank you for your time.

If you have any questions about the study, please contact Cristina Risco at crisco1@umd.edu or Ruth Fassinger at rfassing@umd.edu. This study has been approved by the University of Maryland Institutional Review Board (IRB). 


\section{APPENDIX E: INFORMED CONSENT}

\begin{tabular}{|c|c|}
\hline Project Title & $\begin{array}{l}\text { Evaluation of a Culturally Inclusive Model of Sexual Minority } \\
\text { Identity Formation }\end{array}$ \\
\hline $\begin{array}{l}\text { Why is this } \\
\text { research being } \\
\text { done? }\end{array}$ & $\begin{array}{l}\text { This is a research project being conducted by Ruth Fassinger, Ph.D. } \\
\text { and Cristina Risco, B.S. at the University of Maryland, College } \\
\text { Park. We are inviting you to participate in this research project } \\
\text { because you identify as a racial/ethnic minority and/or person of } \\
\text { color with a same-sex orientation or attraction. The purpose of this } \\
\text { research project is to learn more about the experiences of sexual } \\
\text { minority identification for persons of color. }\end{array}$ \\
\hline $\begin{array}{l}\text { What will I be } \\
\text { asked to do? }\end{array}$ & $\begin{array}{l}\text { Participating in this study involves being asked to complete several } \\
\text { questionnaires concerning your particular life experiences with } \\
\text { sexual minority identification. Participation in the study will take } \\
\text { approximately } 20-30 \text { minutes. You are free to withdraw your } \\
\text { consent to participate and may discontinue your participation in the } \\
\text { study at any time without consequence. After submitting this form, } \\
\text { you will be connected to the survey. Survey items refer to your } \\
\text { experience with the process of sexual minority identification, group } \\
\text { orientation, self-disclosure, belief structure, and demographic } \\
\text { information. After completing the survey, you will have the } \\
\text { opportunity to enter a drawing for one of two } 50 \$ \text { gift cards to } \\
\text { Target. The entry form requires an email address; however the form } \\
\text { is disconnected from your survey. Entry into the drawing is } \\
\text { completely voluntary. }\end{array}$ \\
\hline $\begin{array}{l}\text { What about } \\
\text { confidentiality? }\end{array}$ & $\begin{array}{l}\text { We will do our best to keep your personal information confidential. } \\
\text { To help protect your confidentiality, we will not include identifiable } \\
\text { information on data files. All computer files will be password- } \\
\text { protected. However, due to the public nature of the internet, absolute } \\
\text { confidentiality cannot be guaranteed. The possibility of someone } \\
\text { intercepting your data is highly unlikely, although possible. If you } \\
\text { do not exit or close your internet browser when you have completed } \\
\text { your survey it is possible that another person using your computer at } \\
\text { a later time could view your responses. It is therefore important that } \\
\text { you exit your browser after you have submitted your survey. At the } \\
\text { end of the survey, you will be given the opportunity to enter a } \\
\text { drawing to win one of two } 50 \$ \text { gift cards to Target. Participation in } \\
\text { this drawing is completely voluntary; although you must enter an } \\
\text { email address to participate in the drawing, you do not need to enter } \\
\text { your email address to participate in the survey. Your survey } \\
\text { responses will not be linked to your email address; this information } \\
\text { will be stored in two different data files. Email addresses entered for } \\
\text { participation in the drawing will be kept in the researcher's } \\
\text { password-protected file and will be destroyed immediately following } \\
\text { the drawing. }\end{array}$ \\
\hline What are the & There may be some risks from participating in this research study. \\
\hline
\end{tabular}




\begin{tabular}{|c|c|}
\hline $\begin{array}{l}\text { risks of this } \\
\text { research? }\end{array}$ & $\begin{array}{l}\text { Your participation in this survey could elicit uncomfortable feelings } \\
\text { (e.g., prompt you to reflect on negative experiences with disclosure } \\
\text { of identity). }\end{array}$ \\
\hline $\begin{array}{l}\text { What are the } \\
\text { benefits of this } \\
\text { research? }\end{array}$ & $\begin{array}{l}\text { This research is not designed to help you personally, but the results } \\
\text { may help the investigators learn more about the experiences of } \\
\text { sexual minority identification for persons of color. It is our hope that } \\
\text { other people might benefit from this study through improved } \\
\text { understanding of diverse experiences with the process of self- } \\
\text { identification. }\end{array}$ \\
\hline $\begin{array}{l}\text { Do I have to be } \\
\text { in this research? } \\
\text { May I stop } \\
\text { participating at } \\
\text { any time? }\end{array}$ & $\begin{array}{l}\text { Your participation in this research is completely voluntary. You may } \\
\text { choose not to take part at all. If you decide to participate in this } \\
\text { research, you may withdraw your participation at any time. If you } \\
\text { decide not to participate in this study or if you stop participating at } \\
\text { any time, you will not be penalized. However, only those who } \\
\text { complete the survey will be given the option to enter their email } \\
\text { address to participate in the drawing. }\end{array}$ \\
\hline $\begin{array}{l}\text { What if I have } \\
\text { questions? }\end{array}$ & $\begin{array}{l}\text { This research is being conducted by Ruth Fassinger, Ph.D. and } \\
\text { Cristina Risco, B.S. at the University of Maryland, College Park. If } \\
\text { you have any questions about the research study itself, please } \\
\text { contact Cristina Risco at crisco1@ umd.edu or Ruth Fassinger at } \\
\text { rfassing@ umd.edu. If you have questions about your rights as a } \\
\text { research participant, please contact: Institutional Review Board } \\
\text { Office, University of Maryland, College Park, Maryland, 20742; } \\
\text { (e-mail) irb@deans.umd.edu; (telephone) 301-405-0678. This } \\
\text { research has been reviewed according to the University of Maryland, } \\
\text { College Park IRB procedures for research involving human subjects. }\end{array}$ \\
\hline \multirow[t]{3}{*}{$\begin{array}{l}\text { Statement of Age } \\
\text { of Subject and } \\
\text { Consent: }\end{array}$} & $\begin{array}{l}\text { Your acceptance indicates that: you are at least } 18 \text { years of age; the } \\
\text { research has been explained to you; your questions have been fully } \\
\text { answered; and you freely and voluntarily choose to participate in this } \\
\text { research project. }\end{array}$ \\
\hline & $\begin{array}{l}\text { IRB APPROVED } \\
\text { EXPIRES ON }\end{array}$ \\
\hline & $\begin{array}{l}\text { UNIVERSTTY OFMARYLAND } \\
\text { COLLEGEPARK }\end{array}$ \\
\hline
\end{tabular}




\section{APPENDIX F: SURVEY}

This is a study of the life experiences of People of Color with Same-Sex Attractions. The questions in this survey apply to you if you identify as a person of color or racial/ethnic minority and as any of the following: lesbian, gay, bisexual, queer, questioning, bi-curious, same-gender loving, exploring same-sex sexuality, or have some same-sex attraction or orientation.

Throughout the survey, questions are intended to refer to same-sex orientations or attractions. The terms GBQ and LGBQ are used as short hand to denote same-sex orientations or attractions (e.g., "GBQ men" means men who are same-sex oriented or same-sex attracted).

\section{DEMOGRAPHIC QUESTIONNAIRE}

Instructions: Please provide the following information about yourself.

1. Age:

2. Highest level of education that you have COMPLETED:

Elementary School

Middle/Junior High School

High School/GED

Some College/Technical School/Community College

4 year College

Some Professional/Graduate School

Professional/Graduate School

3. If you responded Professional/Graduate school, please specify type of degree (e.g., MA/MS, MD, PhD, EdD, DVM, etc.):

4. Student Status:

High School student

Undergraduate student

Graduate student

Not a student

Other, please specify

5. Employment Status:

Work full time

Work part time

Not employed

6. Your annual income:

Less than $\$ 12,500$

$\$ 12,500$ to $\$ 24,999$ 
$\$ 25,000$ to $\$ 49,999$

$\$ 50,000$ to $\$ 74,999$

$\$ 75,000$ to $\$ 99,999$

$\$ 100,000$ to $\$ 149,999$

$\$ 150,000$ to $\$ 174,999$

$\$ 175,000$ and over

I don't know

7. I would identify my social class as:

8. Race/Ethnicity (please select all that apply):

African American/Black

Asian American/Pacific Islander

Middle Eastern

Native American/American Indian

Hispanic/Latina/o/Chicano

White/Caucasian (not of Hispanic/Latina/o origin)

Multi-racial

Other, please specify

9. If Multi-racial, please specify:

10. If Hispanic/Latina/o, please specify (i.e., Chicana/o, Cuban American, Mexican American, Puerto Rican, etc.):

11. If Asian American/Pacific Islander, please specify (i.e., Chinese American, Filipino American, Japanese American, etc.):

12. Do you identify as a "person of color?"

Yes

No

13. Do you identify as a "racial/ethnic minority person?"

Yes

No

14. Nationality:

15. Country of Birth:

16. How many years have you live in the United States?

17. In what region of the United States do you live?

Northwest

West Coast

Southwest 
West

Midwest

Northeast

Mid-Atlantic

Southeast

18. Do you live in the Washington DC metropolitan area?

Yes

No

19. Which of the following best describes where you lived most of your life?

Urban area

Suburban area

Small town

Rural Area

20. Which of the following best describes where you live now?

Urban area

Suburban area

Small town

Rural Area

21. Your religion (please select all that apply):

Agnostic

Atheist

Roman Catholic

Greek Orthodox

Protestant

Baptist

Presbyterian

Methodist

Episcopalian

Lutheran

Fundamentalist Christian

Pentecostal

Muslim/Islam

Jewish

Mormon

Hindu

Buddhist

Other, please specify

Gender and Sexuality:

22. In terms of sexual orientation labeling, I refer to myself as:

Lesbian 
Gay

Bisexual

Queer

Questioning

Same-Sex Oriented or Attracted

Other, please specify

23. Please provide any comments you may have about your sexual identity label(s)?

24. Relationship Status:

24a. Single:

Yes

No

Gender of Partner:

NA

Woman

Man

Transgender/Gender-Variant

24b. Dating:

Yes

No

Gender of Partner:

NA

Woman

Man

Transgender/Gender-Variant

24c. Long Term Relationship:

Yes

No

Gender of Partner:

NA

Woman

Man

Transgender/Gender-Variant

24d. Committed/Married:

Yes

No

Gender of Partner:

NA

Woman

Man

Transgender/Gender-Variant

24e. Divorced/Separated:

Yes

No

Gender of Partner: 


\section{NA}

Woman

Man

Transgender/Gender-Variant

24f. Widowed:

Yes

No

Gender of Partner:

NA

Woman

Man

Transgender/Gender-Variant

Gender: Because this survey focuses on same or other-gender feelings and relationships we request that you indicate a gender identification for yourself as man or woman. Please also indicate additional or alternative gender identifications if relevant.

25. Additional or alternative gender identification(s) such as transgender or gendervariant:

26. Primary Gender Identification:

Man

Woman

\section{SAME-SEX ORIENTATION IDENTITY QUESTIONNAIRE_WOMEN}

Instructions: The following items are intended to identify the beliefs and feelings that you have about your sexual identity at this point in time. Some of the items may not apply to you, and some may have applied to you in the past but not the present. Please respond to all items and endorse most strongly the items that capture your feelings about yourself NOW. You may want to scan the items quickly before responding so that you get an idea of how the items differ. Remember to endorse most strongly those items that describe you NOW.

Rating Scale:

$1=$ Disagree strongly

$2=$ Disagree

$3=$ Disagree somewhat

$4=$ Neither disagree nor agree

$5=$ Agree somewhat

$6=$ Agree

$7=$ Agree strongly

Items:

1. I can't stop thinking about the way I have been mistreated because of my same-sex orientation. 
2. My intimacy with women is successfully incorporated into my overall identity.

3. I may be interested in dating women.

4. I want to get to know LGBQ women, but the stigma attached to them is frightening.

5. I fully accept my emotional and sexual connection with women.

6. I get angry a lot at the way straight people talk about and treat LGBQ people.

7. The way I feel about women may mean something.

8. I am interested in women as partners/lovers, just as much or more than I am in men.

9. I have a strong desire to touch another woman's body.

10. I am withdrawing from the straight world.

11. I am aware that many straight people don't even know that LGBQ people exist.

12. I feel a deep contentment about my love of other women.

13. I fully accept and understand that my sexuality makes me a member of a LGBQ community.

14. There may be women out there who have the same kinds of sexual desires that I do.

15. I am no longer interested exclusively in men as intimate partners.

16. I am aware that I feel different from most straight women.

17. My sexuality is an integrated part of my social and public life.

18. I can't even imagine what a room full of LGBQ women and men would be like.

19. I feel pulled toward women in ways I don't understand.

20. Sometimes I get angry at the way LGBQ people are treated, but I'm not consumed by it.

21. I wonder if the way I feel means that I am in love with a woman.

22. There are LGBQ people everywhere, and I can often sense who they are.

23. I could imagine myself living with a female partner/lover.

24 . Heterosexuality may not be all there is.

25. I have a strong desire to kiss another woman.

26. I only feel at ease in LGBQ surroundings.

27. I wonder what it might be like to be romantic with a woman.

28. I have no idea how many women out there are attracted to other women.

29. I might like to be sexual with a woman.

30. I feel more complete as a person because I am consistently doing what I want to do in terms of love and sex.

31. I realize that I have been conditioned to view LGBQ people negatively.

32. I believe there are many straight people who are accepting of LGBQ people.

33. I feel comfortable sexually and emotionally with women.

34. I don't know why I feel nervous and/or emotional around women.

35. My romantic relationships with women are an important part of me, but they are not the only thing that defines me.

36. I am undergoing a personal liberation and becoming involved in a LGBQ culture.

37. I am a person who has or wants intimate romantic relationships with women.

38. Getting to know LGBQ people for the first time is scary but exciting.

39. As a LGBQ woman, I can relate comfortably to both LGBQ and straight people

40. It is very important for me to find and meet LGBQ people.

\section{SAME-SEX ORIENTATION IDENTITY QUESTIONNAIRE_MEN}


Instructions: The following items are intended to identify the beliefs and feelings that you have about your sexual identity at this point in time. Some of the items may not apply to you, and some may have applied to you in the past but not the present. Please respond to all items and endorse most strongly the items that capture your feelings about yourself

NOW. You may want to scan the items quickly before responding so that you get an idea of how the items differ. Remember to endorse most strongly those items that describe you NOW.

Rating Scale:

$1=$ Disagree strongly

$2=$ Disagree

3 = Disagree somewhat

$4=$ Neither disagree nor agree

$5=$ Agree somewhat

$6=$ Agree

$7=$ Agree strongly

Items:

1. I prefer spending time with GBQ men because I find them much more interesting than straight men.

2. My intimacy with men is successfully incorporated into my overall identity.

3. I feel attracted to a specific man, but I'm not sure yet that I would be attracted to other men.

4. I want to get to know GBQ men, but the stigma attached to them is frightening.

5. I love and appreciate myself as a man who is sexually attracted to other men.

6. I get angry a lot at the way straight people talk about and treat LGBQ people.

7. There is something strange about me compared to other straight men.

8. My feelings and/or fantasies are beginning to align with my sexual behavior.

9. I have a strong desire to touch another man's body.

10. I can't stop thinking that some of my suffering could have been avoided if my samesex orientation had been accepted.

11. I am aware that many straight people are disapproving of GBQ men.

12. I feel a deep contentment about my love of other men.

13. I fully accept and understand that my sexuality makes me a member of a GBQ community.

14. There may be men out there who have the same kinds of sexual desires that I do.

15. I am no longer interested exclusively in women as intimate partners.

16. I am aware that I feel different from most straight men.

17. My sexuality is an integrated part of my social and public life

18. I can't even imagine what a room full of GBQ men would be like.

19. I don't seem to like dating women as much as other men do.

20. Sometimes I get angry at the way GBQ men are treated, but I'm not consumed by it.

21. I am interested in being intimate with men.

22. I feel guilty about attitudes I had towards GBQ men in the past.

23. I could imagine myself living with a male partner/lover. 
24. I'm afraid to associate with GBQ men because it might reveal my sexuality to other people

25. I have a strong desire to kiss another man.

26. I feel sad and/or angry that societal prejudice stood in the way of my true feelings for men.

27. It scares me that I am not exclusively attracted to women.

28. I have no idea how many men out there are attracted to other men.

29. I want to become closer to men or to a certain man.

30. I feel more complete as a person because I am consistently doing what I want to do in terms of love and sex.

31. I realize that I have been conditioned to view GBQ men negatively.

32. While some straight men and women are homonegative/homophobic, many are not.

33. I clearly feel comfortable sexually with men.

34. I don't know why I feel nervous and/or emotional around men.

35. My love for men is an important part of me, but it is not the only thing that defines me.

36. I am undergoing a personal liberation and becoming involved in a GBQ culture.

37. I know clearly that I am not straight.

38. I wonder about whether I can fit in as a GBQ man.

39. As a GBQ man, I can relate comfortably to both GBQ and straight men.

40. There are GBQ communities out there, and I want to be a part of one.

IDENTITY CONFUSION (Mohr \& Fassinger, 2000) items 1-4

INTERNALIZED HOMONEGATIVITY (Mohr \& Fassinger, 2000) items 5-9

Instructions: For each of the following statements, select the response that best indicates your experience as a lesbian, gay, bisexual, or queer person (LGBQ).

Rating Scale:

$1=$ Disagree strongly

$2=$ Disagree

$3=$ Disagree somewhat

$4=$ Neither disagree nor agree

$5=$ Agree somewhat

$6=$ Agree

$7=$ Agree strongly

Items:

1. I'm not totally sure that I'm a LGBQ person.

2. I keep changing my mind about my sexual orientation.

3. I can't decide whether I am bisexual or lesbian/gay.

4. I get very confused when I try to figure out my sexual orientation.

5. I would rather be straight if I could.

6. I am glad to be a LGBQ person.

7. LGBQ lifestyles are not as fulfilling as heterosexual lifestyles.

8. I am proud to be part of the LGBQ community. 
9. I wish I were heterosexual.

SAME GROUP ORIENTATION derived from the Same Group Orientation scale of the Multigroup Ethnic Identity Measure (Phinney, 1992)

Instructions: Rate the extent to which each of the following statements describes your experiences of community with lesbian, gay, bisexual, or queer (LGBQ) people.

Rating Scale:

$1=$ Disagree strongly

$2=$ Disagree somewhat

$3=$ Agree somewhat

$4=$ Agree strongly

Items

1. I have spent time trying to find out more about the LGBQ community.

2. I am active in organizations or social groups that include mostly LGBQ people.

3. I have a clear sense of my same-sex orientation/attraction and what it means for me.

4. I think a lot about my how my life will be affected by my LGBQ group membership.

5. I am happy that I am a member of the LGBQ community.

6. I am not very clear about the role of my same-sex orientation in my life.

7. I really have not spent much time trying to learn more about the culture and history of the LGBQ community.

8. I have a strong sense of belonging to the LGBQ community.

9. I understand pretty well what being a part of the LGBQ community means to me, in terms of how to relate to LGBQ people and straight people.

10. In order to learn more about LGBQ culture, I have often talked to other people about LGBQ culture.

11. I have a lot of pride in the LGBQ community and its accomplishments.

12. I participate in LGBQ cultural practices, such as pride events, benefits, or marches.

13. I feel a strong attachment towards the LGBQ community.

14. I feel good about being a part of the LGBQ community.

OUTNESS INVENTORY (Mohr \& Fassinger, 2000)

Instructions: Use the following scale to indicate how open you are about your sexual orientation to the people listed below. Try to respond to all of the items, but leave items blank if they do not apply to you.

Rating Scale:

$1=$ Person definitely does not know about your sexual orientation status.

$2=$ Person might know about your sexual orientation status, but it is never talked about.

$3=$ Person probably knows about your sexual orientation status, but it is never talked about.

$4=$ Person probably knows about your sexual orientation status, but it is rarely talked about. 
$5=$ Person definitely knows about your sexual orientation status, but it is rarely talked about.

$6=$ Person definitely knows about your sexual orientation status, and it is sometimes talked about.

$7=$ Person definitely knows about your sexual orientation status, and it is openly talked about.

Items:

1. Mother

2. Father

3. Siblings (sisters, brothers)

4. Extended family/relatives

5. My new straight friends

6. My work peers

7. My work supervision

8. Members of my religious community (e.g., church, temple)

9. Leaders of my religious community (e.g., minister, rabbi)

10. Strangers, new acquaintances

11. Extended family, relatives

DOGMATISM (Troldahl \& Powell, 1965)

Instructions: Please indicate the extent to which you disagree or agree with the following statements.

Rating Scale:

$1=$ Disagree strongly

$2=$ Disagree

$3=$ Disagree somewhat

$4=$ Agree somewhat

$5=$ Agree

$6=$ Agree strongly

Items:

1. In this complicated world of ours, the only way we can know what is going on is to rely on leaders and experts who can be trusted.

2. My blood boils whenever a person stubbornly refuses to admit she/he is wrong.

3. There are two kinds of people in this world: those who are for the truth and those who are against the truth.

4. Most people just don't know what is good for them.

5. Of all the different philosophies which exist in this world, there is probably only one which is correct.

6. The highest form of government is a democracy and the highest form of democracy is a government run by those who are most intelligent.

7. The main thing in life is for a person to want to do something important. 
8. I'd like it if I could find someone who would tell me how to solve my personal problems.

9. Most of the ideas which get printed nowadays aren't worth the paper they are printed on.

10. Man or Woman on his or her own is a helpless and miserable creature.

11. It is only when a person devotes herself or himself to an ideal or cause that life becomes meaningful.

12. Most people just don't give a "damn" for others.

13. To compromise with our political opponent is dangerous because it usually leads to the betrayal of our own side.

14. It is often desirable to reserve judgment about what's going on until one has had a chance to hear the opinions of those one respects.

15. The present is all too often full of unhappiness. It is only the future that counts.

16. The United States and Russia have just about nothing in common.

17. In a discussion I often find it necessary to repeat myself several times to make sure I am being understood.

18. While I don't like to admit this even to myself, my secret ambition is to become a great person, like Einstein, or Beethoven, or Shakespeare.

19. Even though freedom of speech for all groups is a worthwhile goal, it is unfortunately necessary to restrict the freedom of certain political groups.

20. It is better to be a dead hero than to be a live coward.

Free Response (optional): Please tell us if and how your sexual identity intersects with your cultural identity. We are also interested in any feedback you may have to offer about the survey. Thank you for your participation!

1. How did you find out about the survey? (Please select all that apply):

Email listserv

Networking website (e.g., Facebook, Myspace)

Flyer/Print advertisement

Word of mouth/Email invite

Member of the research team

Other, please specify

2. How well do you read and understand English:

Not Very Well

1

2
3
4

Extremely Well

5

A Lot

5

4. Would you like to provide your email address to enter into a drawing for one of two $\$ 50$ Target gift cards?

Yes/N0 


\section{APPENDIX G: DEBRIEFING}

Thank you very much for taking the time to complete this questionnaire and for your willingness to reveal much personal information. The goal of this study is to learn more about the experiences of sexual minority identification for persons of color. While completing surveys of this nature, participants sometimes feel discomfort as they are asked to reflect on sensitive experiences and may wish to seek information or talk with someone. There are numerous counseling services available for both students and members of the community should you wish to speak with someone about your concerns. If you are a student, you may want to contact student mental health services at your institution. If you are not a student, there are community resources that may be of service to you. Below is a list of websites that offer support and information about local community resources. You may also contact the researchers, Cristina Risco at crisco1@umd.edu or Ruth Fassinger at rfassing@umd.edu, for further information about mental health referrals or questions about the study. Results of the study will be made available to you upon request (contact Cristina Risco at crisco1@ umd.edu). Questions or concerns about your rights as a research participant may be directed to the UMD IRB office at irb@deans.umd.edu; phone 301-405-0678.Thank you again!

American Civil Liberties Union_LGBT Project

www.aclu.org/lgbt

GLBT National Help Center/Hotline

www.glnh.org

1-888-THE-GLNH

GLBT Student Pride

www.glbtstudentpride.com

Human Rights Campaign

www.hrc.org 


\section{APPENDIX H: SSOIQ SCORING INSTRUCTIONS}

There are five (5) items for each of eight (8) positions in this sexual minority identity development model. Four (4) phases relate to Individual Sexual identity Development and four (4) phases relate to Group Membership Identity Development:

Phase 1:

Awareness

Phase 2:

Exploration

Phase 3:

Deepening/

Commitment

Phase 4:

Internalization/

Synthesis

Items $8,15,23,33,37$

Items $2,5,12,30,35$

\author{
Individual \\ Sexual Identity
}

Items 7, 16, 19, 27, 34

Items 3, 9, 21, 25, 29

Group Membership

Identity

Items 4, 14, 18, 24, 28

Items $11,22,31,38,40$

Items 1, 6, 10, 26, 36

Items 13, 17, 20, 32, 39

There are two approaches to scoring the SSOIQ:

1.) Each respondent receives a separate mean score for each of the eight subscales. If desired, summed scores also can be used.

2.) To determine the predominant phase for Individual Sexual Identity Development, sum the 5 items for each phase and divide by 5 to obtain a Mean score for that phase; the largest Mean score indicates the predominant phase. Repeat the process on the other branch of the model to determine the predominant phase for Group Membership Identity Development. Note that locations in different phases of the model for each branch are not uncommon. 


\section{References}

Akerlund, M., \& Cheung, M. (2000). Teaching beyond the deficit model: Gay and lesbian issues among African Americans, Latinos, and Asian Americans. Journal of Social Work Education, 36, 279-292.

Alquijay, M. A. (1997). The relationship among self-esteem, acculturation, and lesbian identity formation in Latina lesbians. In B. Greene (Ed.), Ethnic and cultural diversity among lesbians and gay men (vol. 3). Thousand Oaks: Sage Publications.

Anderson, M. L., \& Hill Collins, P. (Eds.) (1998). Race, class, and gender: An anthology. Belmont, CA: Wadsworth.

Bieschke, K.J., Hardy, M.A., Fassinger, R.E., \& Croteau, J.M. (in press). Intersecting identities of gender-transgressive sexual minorities: Toward a new paradigm of affirmative psychology. In B. Walsh (Ed)., Biennial Review of Counseling Psychology. NY: Routledge.

Bieschke, K. J., Paul, P. L., \& Blasko, K. A. (2007). Review of empirical research focused on the experience of lesbian, gay, and bisexual clients in counseling and psychotherapy. In K. J. Bieschke, R. M. Perez, \& K. A. DeBord (Eds.), Handbook of counseling and psychotherapy with lesbian, gay, bisexual, and transgender clients (2 $2^{\text {nd }}$ ed., pp. 293-316). Washington, DC: American Psychological Association.

Bohan, J. S. (1996). Psychology and sexual orientation: Coming to terms. NY: Routledge. 
Brown, L. S. (1995). Lesbian identities: Concepts and issues. In A. R. D’Augelli \& C. J. Patterson (Eds.), Lesbian, gay, and bisexual identities over the lifespan: Psychological perspectives (pp. 3-23). New York: Oxford University Press.

Cass, V. C. (1979). Homosexual identity formation: A theoretical model. Journal of Homosexuality, 4, 219-235.

Cass, V. C. (1984). Homosexual identity formation: Testing a theoretical model. Journal of Sex Research, 20, 143-167.

Chan, C. S. (1995). Issues of sexual identity in an ethnic minority: The case of Chinese American lesbians, gay men, and bisexual people. In A. R. D’Augelli \& C. J. Patterson (Eds.), Lesbian, gay, and bisexual identities over the lifespan: Psychological perspectives (pp. 87-101). New York: Oxford University Press.

Chan, C. (1997). Don't ask, don't tell, don't know: The formation of a homosexual identity and sexual expression among Asian American lesbians. In B. Greene (Ed.), Ethnic and cultural diversity among lesbians and gay men (vol. 3). Thousand Oaks: Sage Publications.

Chapman, B. E., \& Brannock, J. C. (1987). Proposed model of lesbian identity development: An empirical examination. Journal of Homosexuality, 14, 69-80.

Chivers, M. L., Rieger, G., Latty, E., \& Bailey, M. (2004). A sex difference in the specificity of sexual arousal. Psychological Science, 15, 736-744.

Chung, Y. B., \& Katayama, M. (1996). Assessment of sexual orientation in lesbian/gay/bisexual studies. Journal of Homosexuality, 30, 49-62.

Cohen, J. (1988). Statistical power analysis for the behavioral sciences (2nd ed.). Hillsdale, NJ: Lawrence Earlbaum. 
Cohen, J. (1994). The earth is round ( $\mathrm{p}<.05)$. American Psychologist, 49, 997-1003.

Cohen, C. J., \& Jones, T. (1999). Fighting homophobia versus challenging heterosexism: "The failure to transform" revisited. In E. Brandt (Ed.), Dangerous liaisons: Blacks, gays, and the struggle for equality (pp. 80-101). New York: The New Press.

Coleman, E. (1982). Developmental stages of the coming out process. In J. Gonsiorek (Ed.), Homosexuality and psychotherapy: A practitioner's handbook of affirmative models (pp. 31-44). New York: Haworth Press.

Cox, S., \& Gallois, C. (1996). Gay and lesbian identity development: A social identity perspective. Journal of Homosexuality, 30, 1-29.

Croom, G. L. (1999). Lesbian, gay, and bisexual people of color: A challenge to representative sampling in empirical research. In B. Greene \& G. L. Croom (Eds.), Education, research, and practice in lesbian, gay, bisexual and transgender psychology. Thousand Oaks: Sage Publications.

Croteau, J. M., Bieschke, K. J., Fassinger, R. E. \& Manning, J. L. (in press). Counseling psychology and sexual orientation: History, selective trends, and future directions. In R. M. Perez, K. A. DeBord, \& K. J. Bieschke. (Eds.), Handbook of counseling and psychotherapy with lesbian, gay, and bisexual clients. Washington, DC: American Psychological Association Books.

D’Augelli, A. R. (1994). Identity development and sexual orientation: Toward a model of lesbian, gay, and bisexual development. In E. J. Trickett \& R. J. Watts \& D. Birman (Eds.), Human diversity: Perspectives on people in context (pp. 312-333). San Francisco: Jossey-Bass. 
DeVellis, R. F. (1991). Scale development: Theory and applications. Newbury Park, CA: Sage.

Diamond, L. M. \& Savin-Williams, R. (2000). Explaining diversity in the development of same-sex sexuality among young women. Journal of Social Issues, 56, 297.

Dubé, E. M., \& Savin-Williams, R. C. (1999). Sexual identity development among ethnic sexual-minority male youths. Developmental Psychology, 35, 1389-1399.

Eliason, M. J. (1996). Identity formation for lesbian, bisexual, and gay persons: Beyond a “minoritizing" view. Journal of Homosexuality, 30, 31-57.

Enns, C. Z. (2004). Feminist theories and feminist psychotherapies: Origins, themes, and diversity ( $2^{\text {nd }}$ ed). New York: The Haworth Press

Faderman, L. (1984). The "new gay" lesbians. Journal of Homosexuality, 10, 85-95.

Fassinger, R. E. (1991). The hidden minority: Issues and challenges in working with lesbian women and gay men. The Counseling Psychologist, 19, 157-176.

Fassinger, R. E. (1994). Development and testing of the Attitudes toward Feminism and the Women's Movement (FWM) scale. Psychology of Women Quarterly, 18, $389-402$.

Fassinger, R. E. (2000). Gender \& sexuality in human development: Implications for prevention and advocacy in counseling psychology. In S. D. Brown, \& R. W. Lent (Eds.), Handbook of counseling psychology ( $3^{\text {rd }}$ ed.). New York: Wiley.

Fassinger, R. E. (2001a). Lesbian Identity Questionnaire (Revised). College Park: Unpublished instrument, University of Maryland.

Fassinger, R. E. (2001b). Gay Identity Questionnaire (Revised). College Park: Unpublished instrument, University of Maryland. 
Fassinger, R. E., \& Arseneau, J. R. (2007). 'I'd rather get wet than be under that umbrella:" Differentiating among lesbian, gay, bisexual, and transgender people. In K. J. Bieschke, R. M. Perez, \& K. A. DeBord (Eds.), Handbook of counseling and psychotherapy with lesbian, gay, and bisexual clients $\left(2^{\text {nd }}\right.$ ed.). Washington, DC: American Psychological Association Books.

Fassinger, R. E., \& McCarn, S. R. (1991, March). Embracing our diversity: An inclusive model of lesbian identity development. Paper presented at the annual conference of the Association for Women in Psychology, Hartford, CT.

Fassinger, R. E., \& Miller, B. A. (1996). Validation of an inclusive model of sexual minority identity formation on a sample of gay men. Journal of Homosexuality, 32, 53-78.

Firestein, B. A. (2007). Cultural and relational contexts of bisexual women: Implications for therapy. In K. J. Bieschke, R. M. Perez, \& K. A. DeBord (Eds.), Handbook of counseling and psychotherapy with lesbian, gay, bisexual, and transgender clients ( $2^{\text {nd }}$ ed., pp. 91-117). Washington, DC: American Psychological Association.

Fouad, N. A. \& Brown, M. T. (2000). Role of race and social class in development: Implications for counseling psychology. In S. D. Brown, \& R. W. Lent (Eds.), Handbook of counseling psychology ( $3^{\text {rd }}$ ed.). New York: Wiley.

Fukuyama, M. A. \& Ferguson, A. D. (2000). Lesbian, gay, and bisexual people of color: Understanding cultural complexity and managing multiple oppressions. In R. M. Perez, K. A. DeBord, \& K. J. Bieschke. (Eds.), Handbook of counseling and psychotherapy with lesbian, gay, and bisexual clients. Washington, DC: American Psychological Association Books. 
González, F. J. \& Espín, O. M. (1996). Latino men, Latina women, and homosexuality. In R. P. Cabaj, \& T. S. Stein. (Eds.), Textbook of homosexuality and mental health. (pp. 583-601). Washington, DC: American Psychiatric Association.

Gonsiorek, J. C. (1995). Gay male identities: Concepts and issues. In A. R. D’Augelli \& C. J. Patterson (Eds.), Lesbian, gay, and bisexual identities over the lifespan: Psychological perspectives (pp. 24-47). New York: Oxford University Press.

Greene, B. (1994). Ethnic-minority lesbians and gay men: Mental health and treatment issues. Journal of Consulting and Clinical Psychology, 62, 243-251.

Greene, B. (1997). Ethnic minority lesbians and gay men. In B. Greene (Ed.), Ethnic and cultural diversity among lesbians and gay men (pp. 216-239). London: Sage.

Greene, B. (2000). African American lesbian and bisexual women. Journal of Social Issues, 56, 239-249.

Harper, G. W., Jernewall, N., Zea, M. C. (2004). Giving voice to emerging science and theory for lesbian, gay, and bisexual people of color. Cultural Diversity and Ethnic Minority Psychology, 10, 187-199.

Helms, J. E. (1990). Black and White racial identity. Westport, CT: Praeger.

Helms, J. E. (1992). A race is a nice thing to have. Topeka, KS: Content Communications.

Holmes, R. (2001). The relationship of race and gender to adult gay and lesbian identity development. Unpublished master's thesis, University of Maryland, College Park, Maryland.

Johns, D. J. \& Probst, T. M. (2004). Sexual minority identity formation in an adult population. Journal of Homosexuality, 47, 81-90. 
Kahn, J. H. (2006). Factor analysis in counseling psychology research, training, and practice: Principles, advances, and applications. The Counseling Psychologist, 34, 684-718.

Liddle, B. J. (2007). Mutual bonds: Lesbian women's lives and communities. In K. J. Bieschke, R. M. Perez, \& K. A. DeBord (Eds.), Handbook of counseling and psychotherapy with lesbian, gay, bisexual, and transgender clients $\left(2^{\text {nd }}\right.$ ed., pp. 51-70). Washington, DC: American Psychological Association.

Loiacano, D. K. (1989). Gay identity issues among black Americans: racism, homophobia, and the need for validation. Journal of Counseling and Development, 68, 21-25.

Manalansan, M. F., IV. (1994). Searching for community: Filipino gay men in New York City. Amerasia Journal, 20, 59-73.

Martinez, D. G., \& Sullivan, S. G. (1998). African American gay men and lesbians: Examining the complexity of gay identity development. Journal of Human Behavior in the Social Environment, 1, 243-264.

McCarn, S. R. (1991). Validation of a model of sexual minority (lesbian) identity development. Unpublished master's thesis, University of Maryland at College Park.

McCarn, S. R. \& Fassinger, R. E. (1996). Revisioning sexual minority identity formation: A new model of lesbian identity and its implications for counseling and research. The Counseling Psychologist, 24, 508-534. 
McEwen, M. K. (1996). New perspectives on identity development. In S.R. Komives \& D.B. Woodward, Jr. (Eds.), Student services: A handbook for the profession $\left(3^{\text {rd }}\right.$ ed., pp. 188-217). San Francisco: Jossey-Bass.

Meyer, I. H. (1995). Minority stress and mental health in gay men. Journal of Health and Social Behavior, 36, 38-56.

Meyer, I. H. (2003). Prejudice, social stress, and mental health in lesbian, gay, and bisexual populations: Conceptual issues and research evidence. Psychological Bulletin, 129, 674-697.

Minton, H. L., \& McDonald, G. J. (1984). Homosexual identity formation as a developmental process. Journal of Homosexuality, 9, 91-104.

Mohr, J. \& Fassinger, R. (2000). Measuring dimensions of lesbian and gay male experience. Measurement and Evaluation in Counseling and Development, 33, 66-90.

Mohr, J. \& Fassinger, R. (2003). Self-acceptance and self-disclosure of sexual orientation in lesbian, gay, and bisexual adults: An attachment perspective. Journal of Counseling Psychology, 50, 482-495.

Morales, E. S. (1990). Ethnic minority families and minority gays and lesbians. Marriage and Family Review, 14, 217-239.

Morris, J. F. (1997). Lesbian coming out as a multidimensional process. Journal of Homosexuality, 33, 1-22.

Morris, J. F. \& Rothblum, E. D. (1999). Who fills out a "lesbian" questionnaire? Psychology of Women Quarterly, 23, 537-557. 
Myers, L. J., Speight, S. L., Highlen, P. S., Cox, C. I., Reynolds, A. R., Adams, E. M., \& Hanley, C. P. (1991). Identity development and worldview: Toward and optimal conceptualization. Journal of Counseling and Development, 70, 54-63.

Parks, C. A., Hughes, T. L., Matthews, A. K. (2004). Race/ethnicity and sexual orientation: Intersecting identities. Cultural Diversity and Ethnic Minority Psychology, 10, 241-254.

Pedhazur, E. J. (1997). Multiple regression in behavioral research: Explanation and prediction ( $3^{\text {rd }}$ ed.). Belmont, CA: Wadsworth.

Peplau, L. A. (2001). Rethinking women's sexual orientation: An interdisciplinary, relationship-focused approach. Personal Relationships, 8, 1-19.

Perez, R. M., DeBord, K. A., \& Bieschke, K. J. (2000). Handbook of counseling and psychotherapy with lesbian, gay, and bisexual clients. Washington DC: American Psychological Association.

Pett, M.A., Lackey, N. R., \& Sullivan, J. J. (2003). Making sense of factor analysis: The use of factor analysis for instrument development in health care research. Thousand Oaks: Sage Publications.

Phinney, J. S. (1992). The Multigroup Ethnic Identity Measure: A new scale for use with diverse groups. Journal of Adolescent Research, 7, 156-176.

Porter, J. D. (1998). The contribution of gay and lesbian identity development to transformational leadership self-efficacy (Doctoral dissertation, University of Maryland, 1998). Dissertation Abstracts International, 59, 06A.

Potoczniak, D. J. (2007). Development of bisexual men's identities and relationships. In K. J. Bieschke, R. M. Perez, \& K. A. DeBord (Eds.), Handbook of counseling and 
psychotherapy with lesbian, gay, bisexual, and transgender clients ( $2^{\text {nd }}$ ed., $119-$ 146). Washington, DC: American Psychological Association.

Quintana, S. M., Troyano, N., \& Taylor, G. (2001). Cultural validity and inherent challenges in quantitative methods for multicultural research. In J. G. Ponterotto, J. M. Casas, L. A. Suzuki, \& C. M. Alexander (Eds.), Handbook of multicultural counseling ( $2^{\text {nd }}$ ed., pp. 604-630). Thousand Oaks: Sage Publications.

Reynolds, A.L. \& Hanjorgiris, W.F. (2000). Coming out: Lesbian, gay, and bisexual identity development. In R. M. Perez, K. A. DeBord, \& K. J. Bieschke. (Eds.), Handbook of counseling and psychotherapy with lesbian, gay, and bisexual clients. Washington, DC: American Psychological Association Books.

Reynolds, A. I., \& Pope, R. L. (1991). The complexities of diversity: Exploring multiple oppressions. Journal of Counseling and Development, 70, 174-180.

Rokeach, M. (1956). Political and religious dogmatism: An alternative to the authoritarian personality. Psychological Monographs: General and Applied, 70, $1-43$.

Rosario, M., Schrimshaw, E. W., \& Hunter, J. (2004). Ethnic/racial differences in the coming-out process of gay, lesbian, and bisexual youths: A comparison of sexual identity development over time. Cultural Diversity and Ethnic Minority Psychology, 10, 215-228.

Savin-Williams, R. C. (1996). Ethnic- and sexual-minority youth. In R. C. SavinWilliams \& K. M. Cohen (Eds.), The lives of lesbians, gays, and bisexuals: Children to adults (pp. 152-165). New York: Harcourt Brace. 
Savin-Williams, R. C. (2005). The new gay teenager. Cambridge, MA: Harvard University Press.

Savin-Williams, R. C., \& Diamond, L. M. (2000). Sexual identity trajectories among sexual minority youths: Gender comparisons. Archives of Sexual Behavior, 29, 607.

Smith, A. (1997). Cultural diversity and the coming-out process: Implications for clinical practice. In B. Greene (Ed.), Ethnic and cultural diversity among lesbians and gay men: Psychological perspectives on lesbian and gay issues (pp. 279-301).

Thousand Oaks: Sage Publications.

Smith, B. (1999). Blacks and gays healing the great divide. In E. Brandt (Ed.), Dangerous liaisons: Blacks, gays, and the struggle for equality (pp. 15-24). New York: The New Press.

Sophie, J. (1985-1986). A critical examination of stage theories of lesbian identity development. Journal of Homosexuality, 12, 39-51.

Tomlinson, M. \& Fassinger, R. E. (2003). Career development, lesbian identity development, and campus climate among lesbian college students. Journal of College Student Development, 44, 845-860.

Troiden, R. R. (1989). The formation of homosexual identities. Journal of Homosexuality, 17, 43-73.

Troldahl, V. C. \& Powell, F. A. (1965). A short-form of the dogmatism scale for use in field studies. Social Forces, 44, 211-214. 
Waldo, C. R. (1999). Working in a majority context: A structural model of heterosexism as minority stress in the workplace. Journal of Counseling Psychology, 46, 218232.

Wilson, B. D. M. \& Miller, R. L. (2002). Strategies for managing heterosexism used among African-American gay and bisexual men. Journal of Black Psychology, 28, 371-391.

Wilton, L., Halkitis, P. N., English, G., Roberson, M. (2005). An exploratory study of barebacking, club drug use, and meanings of sex in Black and Latino gay and bisexual men in the age of AIDS. Journal of Gay and Lesbian Psychotherapy, 9, 49-72.

Worthington, R. L., Navarro, R. L., Bielstein-Savoy, H., \& Hampton, D. (2008).

Development, Reliability, and Validity of the Measure of Sexual Identity Exploration and Commitment (MoSIEC). Developmental Psychology, 44, 22-33.

Zea, M. C., Reisen, C. A., \& Diaz, R. M. (2003). Methodological issues in research with Latino gay and bisexual men. American Journal of Community Psychology, 31, 281-291. 